Louisiana State University

LSU Digital Commons

$1-1-2021$

\title{
Manipulating Phospholipid Vesicles at the Nanoscale: A Transformation from Unilamellar to Multilamellar by an n-Alkyl- poly(ethylene oxide)
}

\author{
Judith U. De Mel \\ Louisiana State University \\ Sudipta Gupta \\ Louisiana State University \\ Lutz Willner \\ Forschungszentrum Jülich (FZJ) \\ Jürgen Allgaier \\ Forschungszentrum Jülich (FZJ) \\ Laura R. Stingaciu \\ Oak Ridge National Laboratory
}

See next page for additional authors

Follow this and additional works at: https://digitalcommons.Isu.edu/chemistry_pubs

\section{Recommended Citation}

De Mel, J., Gupta, S., Willner, L., Allgaier, J., Stingaciu, L., Bleuel, M., \& Schneider, G. (2021). Manipulating Phospholipid Vesicles at the Nanoscale: A Transformation from Unilamellar to Multilamellar by an n-Alkylpoly(ethylene oxide). Langmuir https://doi.org/10.1021/acs.langmuir.0c03302

This Article is brought to you for free and open access by the Department of Chemistry at LSU Digital Commons. It has been accepted for inclusion in Faculty Publications by an authorized administrator of LSU Digital Commons.

For more information, please contact ir@lsu.edu. 


\section{Authors}

Judith U. De Mel, Sudipta Gupta, Lutz Willner, Jürgen Allgaier, Laura R. Stingaciu, Markus Bleuel, and Gerald J. Schneider 


\section{Manipulating Phospholipid Vesicles at the Nanoscale: A Transformation from Unilamellar to Multilamellar by an $n$-Alkyl- poly(ethylene oxide)}

Judith U. De Mel,* Sudipta Gupta,* Lutz Willner, Jürgen Allgaier, Laura R. Stingaciu, Markus Bleuel, and Gerald J. Schneider*

Cite This: Langmuir 2021, 37, 2362-2375

Read Online

ACCESS | Lلll Metrics \& More | 国 Article Recommendations | st Supporting Information

ABSTRACT: We investigated the influence of an $n$-alkyl-PEO polymer on the structure and dynamics of phospholipid vesicles. Multilayer formation and about a $9 \%$ increase in the size in vesicles were observed by cryogenic transmission electron microscopy (cryo-TEM), dynamic light scattering (DLS), and small-angle neutron/X-ray scattering (SANS/SAXS). The results indicate a change in the lamellar structure of the vesicles by a partial disruption caused by polymer chains, which seems to correlate with about a $30 \%$ reduction in bending rigidity per unit bilayer, as revealed by neutron spin echo (NSE) spectroscopy. Also, a strong change in lipid tail relaxation was observed. Our results point to opportunities using synthetic polymers to control the structure and dynamics of membranes, with possible applications in technical materials and also in drug and nutraceutical delivery.

\section{INTRODUCTION}

Phospholipid vesicles are a versatile system for a variety of applications. ${ }^{1-3}$ Primarily made up of phospholipids that have a polar headgroup and hydrophobic tails, these are spherical self-assemblies that can span sizes from tens of nanometers to several micrometers. Despite the size differences, the structure is universal for vesicles with an aqueous core encapsulated by a phospholipid bilayer with the polar headgroups of the two layers facing opposite directions, allowing the lipid tails to be sandwiched in the middle. The properties of phospholipid vesicles can be changed using additives such as cholesterol, small molecules, and macromolecules such as synthetic polymers. ${ }^{4-12}$ From the initial stages of incorporating synthetic polymers into phospholipid vesicles, the present vesicle formulations with associated polymers have come a long way in terms of fine tuning their properties for drug delivery and applications in cosmetics, nutraceuticals, and food technology. ${ }^{13}$ Polymer-liposome interactions could lead to (i) coating, (ii) insertion, (iii) disruption, and/or (iv) transforming the entire vesicle self-assembly. ${ }^{14}$ These interactions are susceptible to experimental parameters such as solvent quality, temperature, ionic strength, and pressure and ultimately determine the liposome morphology, bilayer structure, and dynamics in the presence of polymers. Altogether, they can unfold a plethora of benefits in applications when it comes to the (i) encapsulation efficiency, (ii) controlled release, and (iii) drug transport mechanisms

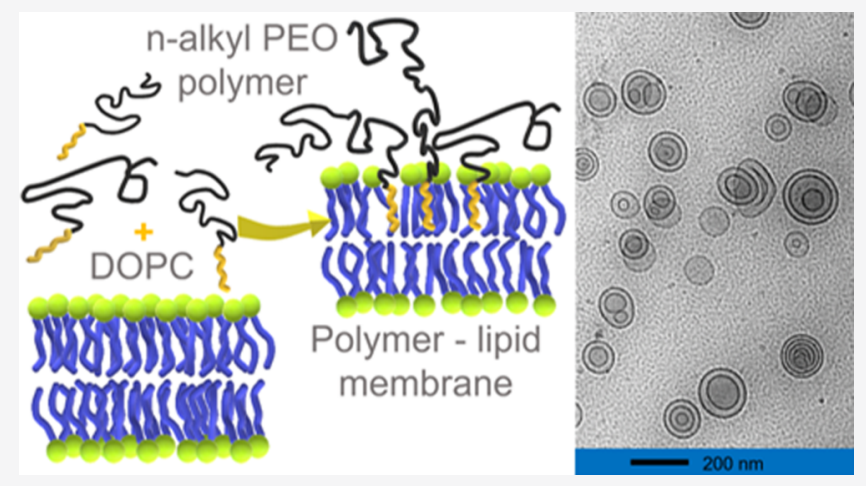

such as skin penetration. ${ }^{15,16}$ Despite the abundance of applications pointing to the importance of molecular-level interactions, fundamental questions such as how polymerliposome interactions on the nanoscale drive the structure and dynamic changes of these self-assemblies are not well understood.

One popular polymer for studying interactions with phospholipid membranes has been PEG (poly(ethylene glycol)), which has a repeating unit identical to that of PEO (poly(ethylene oxide)) or POE (polyoxyethylene), $-\left(\mathrm{CH}_{2}-\right.$ $\left.\mathrm{CH}_{2}-\mathrm{O}-\right)_{n}$. Throughout the article, we will use the abbreviation PEO to be consistent. There are a variety of applications of PEOs from steric stabilization agents ${ }^{17}$ to formulated "stealth" vesicles which increase blood circulation times. $^{18}$ Although the biocompatibility of PEO is well established, higher-molecular-weight PEOs are known to show various levels of cytotoxicity and aggregation. ${ }^{19,20}$ Therefore, we decided on a PEO-based polymer with a low degree of polymerization $(n<100)$ for this work. PEO chains

Received: November 17, 2020

Revised: January 27, 2021

Published: February 11, 2021 
when functionalized with hydrophobic molecules, such as pairs of dodecyl or cholesteryl ends, can anchor to the outer hydrophilic part of the bilayer, while chains form a "mushroom" or "brush" conformation that changes the rigidity of the bilayer. $^{21}$ Extending to longer blocks such as PEO-PPO (poly(propylene oxide)) diblock copolymers or PEO-PPOPEO triblock copolymers, also known as poloxamers, introduce the opportunity to manipulate the liposomes from inside the bilayer and from the aqueous phase., ${ }^{8,9,22}$ Poly(oxyethylene alkyl ethers) $\left(\mathrm{C}_{i} \mathrm{E}_{j}\right)$ or $n$-alkyl PEO polymers are at an intermediate position between $\mathrm{PEO}$ and diblock copolymers such as PEO-PPO. These $n$-alkyl PEO polymers consisting of hydrophilic poly(ethylene oxide) chains connected to simple hydrophobic alkyl chains are nonionic surfactant-like polymers that can show multiple self-assembled structures. ${ }^{23-25}$ These are commonly used in soaps and detergents in combination with other surfactants. Recently, they have also picked up momentum in biomedical applications. ${ }^{26,27}$ Polymers with $n$-alkyl PEO-type structures are extensively used in cosmetics, personal care, and consumer products as emulsifiers and surfactants. The nonionic nature combined with the amphiphilic nature allows these polymers to exhibit unique properties depending on environmental conditions such as the hydrophilic-lipophilic balance (HLB), $\mathrm{pH}$, temperature, ionic strength, and lipid headgroups in interactions. $^{28}$

In previous studies, it has been shown that nonionic surfactants can influence lipid bilayers in numerous ways. ${ }^{29-31}$ For instance, detergents such as Triton X-100 have been shown to modify the ion permeability of lipid membranes. ${ }^{32}$ Similarly, $n$-alkyl PEO polymers which belong to the class of nonionic surfactants can cause several modifications of phospholipid bilayers. The work of Liu et al. has shown that $n$-alkyl PEO polymers can insert into phospholipid bilayers by their hydrophobic end using quartz crystal microbalance (QCM) experiments. ${ }^{10}$ The chain length of the hydrophobic segment is known to play a key role in determining the successful insertion into the bilayer. ${ }^{8,33}$ In the work by Gutberlet et al. using POPC and $\mathrm{C}_{12} \mathrm{E}_{n}(n \leqslant 6,8)$ systems, they have shown that the molar ratio of polymeric surfactant can decrease the thickness of the bilayer and gradually transform the system to different micellar structures. ${ }^{11}$

This study investigates the structural and dynamic changes of the lipid membrane caused by blending aqueous solutions of poly(ethylene oxide)-mono- $n$-octadecyl ether, $\mathrm{C}_{18}$-PEO4, and DOPC (1,2-dioleoyl-sn-glycero-3-phosphatidylcholine). This implies that $\mathrm{C}_{18}-\mathrm{PEO} 4$ is added to the solution of liposomes. $\mathrm{C}_{18}$-PEO4 consists of an $n$-octadecyl hydrophobic alkyl tail and a $\sim 4 \mathrm{~kg} / \mathrm{mol}$ hydrophilic PEO chain (PEO4 4000 g/mol). Similar polymers have been shown to play key roles in interactions with membranes for integral proteins ${ }^{34,35}$ and to slow lipid and vitamin $\mathrm{E}$ oxidation. ${ }^{36}$ These polymers are known to form stable micellar structures in aqueous solutions. $^{37,38}$ We determined the influence of $\mathrm{C}_{18}$-PEO4 polymers on the nanoscale structure and dynamics of phospholipid membranes, which is useful for a fundamental understanding of vesicle self-assembly and enhancing drugs or nutraceutical delivery.

\section{THEORETICAL BACKGROUND}

In this section, we present the data modeling theory used to understand the macroscopic scattering cross section, $\mathrm{d} \Sigma / \mathrm{d} \Omega$, for the vesicle structure and the membrane. Both the SANS and SAXS experiments are performed at an ambient temperature of $20{ }^{\circ} \mathrm{C}$, which corresponds to the fluid phase of DOPC. $^{39}$

Vesicle Structure. A modified core-shell model is used to describe the vesicle form factor. ${ }^{40-42}$ As illustrated in Figure $\mathrm{S} 1$, the core is filled with water which is encapsulated by $N$ shells of lipids and $N-1$ layers of solvent in the case of multilayer vesicles (MLVs). The thickness and scatteringlength density of each shell are assumed to be identical. The corresponding form factor is given by

$$
P(Q, R, t, \Delta \rho)=\frac{\phi[F(Q)]^{2}}{V\left(R_{N}\right)}
$$

with

$$
\begin{gathered}
F(Q)=\left(\rho_{\text {shell }}-\rho_{\text {solv }}\right) \sum_{i=1}^{N}\left[3 V\left(r_{i}\right) \frac{\sin \left(Q r_{i}\right)-Q r_{i} \cos \left(Q r_{i}\right)}{\left(Q r_{i}\right)^{3}}\right. \\
\left.-3 V\left(R_{i}\right) \frac{\sin \left(Q R_{i}\right)-Q r_{i} \cos \left(Q R_{i}\right)}{\left(Q R_{i}\right)^{3}}\right]
\end{gathered}
$$

For

$$
\begin{array}{ll}
r_{i}=r_{\mathrm{c}}+(i-1)\left(t_{\mathrm{s}}+t_{\mathrm{w}}\right) & \text { solvent radius before shell } i \\
R_{i}=r_{i}+t_{\mathrm{s}} & \text { shell radius for shell } i
\end{array}
$$

Here, $V(r)$ is the volume of the sphere with radius $r, r_{\mathrm{c}}$ is the radius of the core, $t_{\mathrm{s}}$ is the thickness of the individual shells, $t_{\mathrm{w}}$ is the thickness of the interbilayer water, and $\phi$ is the corresponding lipid volume fraction. The outer perimeter radius is given by $R_{\text {SANS }}=r_{\mathrm{c}}+N t_{\mathrm{s}}+(N-1) t_{\mathrm{w}}$. For DOPC, we used the neutron scattering-length density (NSLD) of the shell, $\rho_{\text {shell }}=3.01 \times 10^{9} \mathrm{~cm}^{-2}$, and for $\mathrm{D}_{2} \mathrm{O}$, we used the scattering-length density of the solvent, $\rho_{\text {solv }}=6.36 \times 10^{10}$ $\mathrm{cm}^{-2}$. ${ }^{43}$ The macroscopic scattering cross section is obtained from the scattering intensity in SANS and is given by

$$
\begin{aligned}
\frac{\mathrm{d} \Sigma}{\mathrm{d} \Omega}(Q)_{\text {SANS }} & =I(Q)_{\text {SANS }} \\
& =\int \mathrm{d} r P\left(Q, R, t, \rho_{\text {lipo }}, \rho_{\text {solv }}\right) s(r)
\end{aligned}
$$

For the size polydispersity, $s(r)$, we used a Schulz distribution and a log-normal distribution. In addition, the thicknesses of the shell and the solvent are convoluted with a Gaussian distribution function to account for the thickness polydispersity.

Membrane Structure. The random lamellar sheet consisting of the heads and tails of the phospholipids can be modeled using the Caille structure factor. ${ }^{44-46}$ SAXS provides direct access to the macroscopic scattering cross section given by the scattering intensity, and for a random distribution of the lamellar phase, it is given by

$$
\frac{\mathrm{d} \Sigma}{\mathrm{d} \Omega}(Q)_{\mathrm{SAXS}}=I(Q)_{\mathrm{SAXS}}=2 \pi \frac{V P(Q) S(Q)}{Q^{2} d}
$$

with the scattering volume, $V$, and the distance to the lamellae, $d$. The form factor is given by

$$
\begin{aligned}
& P(Q)=\frac{4}{Q^{2}} \\
& \quad\left[\Delta \rho_{\mathrm{H}}\left\{\sin \left(Q\left(\delta_{\mathrm{H}}+\delta_{\mathrm{T}}\right)\right)-\sin \left(Q \delta_{\mathrm{T}}\right)\right\}+\Delta \rho_{\mathrm{T}} \sin \left(Q \delta_{\mathrm{T}}\right)\right]^{2}
\end{aligned}
$$


The scattering contrasts for the head and tail are $\Delta \rho_{\mathrm{H}}$ and $\Delta \rho_{\mathrm{T}}$, respectively. The corresponding thicknesses are $\delta_{\mathrm{H}}$ and $\delta_{\mathrm{T}}$, respectively, as presented in Figure S1. The head-to-head bilayer thickness is given by $\delta_{\mathrm{HH}}=2\left(\delta_{\mathrm{H}}+\delta_{\mathrm{T}}\right)$. The Caille structure factor is given by

$$
S(Q)=1+2 \sum_{n=1}^{N-1}\left(1-\frac{n}{N}\right) \cos (Q d n) \exp \left(-\frac{2 Q^{2} d^{2} \alpha(n)}{2}\right)
$$

with the number of lamellar layers, $N$, and the correlation function for the lamellae, $\alpha(n)$, defined by

$$
\alpha(n)=\frac{\eta_{\mathrm{cp}}}{4 \pi^{2}}\left(\ln (\pi n)+\gamma_{\mathrm{E}}\right)
$$

with $\gamma_{\mathrm{E}}=0.57721$ being Euler's constant. The elastic constant for the membranes is expressed in terms of the Caille parameter, $\eta_{\mathrm{cp}}=\frac{\mathrm{Q}_{0}{ }^{2} k_{\mathrm{B}} T}{8 \pi \sqrt{\kappa_{\mathrm{b}} \kappa_{\mathrm{A}} / \delta_{\mathrm{HH}}}}$, where $\kappa_{\mathrm{b}}$ and $\kappa_{\mathrm{A}}$ are the bending elasticity and the compression modulus of the membranes, respectively. Here, $\kappa_{\mathrm{A}}$ is associated with the interactions between the membranes. The position of the first-order Bragg peak is given by $Q_{0}, k_{\mathrm{B}}$ is the Boltzmann constant, and $T$ is the absolute temperature.

Vesicle Dynamics. Neutron spin echo (NSE) spectroscopy has proven to be a powerful tool for following the molecular motions in vesicles. ${ }^{47-49}$ This method reaches the highest energy resolution $(\sim n \mathrm{eV})$ of all available neutron scattering spectroscopy techniques and allows us to measure the dynamic structure factor or the intermediate scattering function, $S(Q, t)$, for up to several hundred nanoseconds.

Recently, it has been shown that diffusion, membrane fluctuations, the confined motion of lipid tails, and translational diffusion influence $S(Q, t) .^{50}$

$$
\begin{gathered}
S_{\text {liposome }}(Q, t)=\left(n_{\mathrm{H}, \text { head }}+n_{\mathrm{H}, \mathrm{tail}}\left(\mathcal{H}(Q)+(1-\mathcal{A}(Q)) \exp \left(-\left(\frac{t}{\tau}\right)^{\beta}\right)\right)\right) \\
S_{\mathrm{ZG}}(Q, t) \exp \left(-D_{\mathrm{t}} Q^{2} t\right)
\end{gathered}
$$

This equation can be divided into three terms: the lipid tail motion, the membrane undulations $S_{\mathrm{ZG}}(Q t)$, and the translational diffusion, $\exp \left(-D_{\mathrm{t}} Q^{2} t\right)$.

Variables $n_{\mathrm{H} \text {,head }}$ and $n_{\mathrm{H} \text {,tail }}$ relate to the relative numbers of protons in the head and the tail, respectively, and represent the contrast. In the case of $h$-DOPC, $n_{\mathrm{H} \text {,head }}=0.21$ and $n_{\mathrm{H} \text {,tail }}=1-$ $n_{\mathrm{H} \text {,head }}=0.79$. Prefactor $\mathcal{A}(\mathrm{Q})$ refers to the elastic fraction of the lipid tail motion and is formally equivalent to the elastic incoherent structure factor (EISF) from quasielastic neutron scattering (QENS). ${ }^{50}$

The membrane undulations can be modeled by the ZilmanGranek (ZG) approach: ${ }^{51}$

$$
S_{\mathrm{ZG}}(Q, t)=A \exp \left[-\left(\Gamma_{Q} t\right)^{2 / 3}\right]
$$

The free parameters are the $Q$-dependent decay rate, $\Gamma_{\mathrm{Q}}$, and the amplitude, $A$.

The effective bending modulus, $\tilde{\kappa}$, is calculated from modified ZG theory by Watson and Brown ${ }^{52}$ as

$$
\frac{\Gamma_{q}}{Q^{3}}=0.025 \gamma \frac{k_{\mathrm{B}} T}{\eta} \sqrt{\frac{k_{\mathrm{B}} T}{\tilde{\kappa}}}
$$

Here, $\gamma$ is a weak monotonously increasing function of $\tilde{\kappa} / k_{\mathrm{B}} T$. Unlike bicontinuous microemulsions, ${ }^{53}$ the effective bending modulus of the lipid membrane is $\frac{\tilde{\kappa}}{k_{\mathrm{B}} T} \gg 1$; therefore, $\gamma=1$ is a reasonable approximation as suggested by Zilman and Granek. ${ }^{51}$ Here, $\eta$ is the solvent viscosity, $k_{\mathrm{B}}$ is the Boltzmann constant, and $T$ is the temperature on an absolute scale.

The effective bending modulus, $\tilde{\kappa}$ (or dynamic curvature modulus), is related to the bilayer curvature modulus, $\kappa_{\eta}$, given by $\tilde{\kappa}=\kappa_{\eta}+2 h^{2} k_{\mathrm{m}} .{ }^{52}$ Here, $\kappa_{\eta}$ is the parameter of interest which can be obtained from NSE. The monolayer area compressibility modulus for uniform plates of monolayers can be related to the monolayer bending rigidity, $\kappa_{\mathrm{b}}$, as $k_{\mathrm{m}}=12 \kappa_{\mathrm{b}} / h^{t^{2}} .{ }^{54}$ Here, $h_{\mathrm{t}}$ is the thickness of the tail-only region of the membrane (monolayer hydrocarbon thickness) and $h$ is the monolayer thickness or the height of the neutral surface from the bilayer midplane given by $h=\delta_{\mathrm{HH}} / 2$. To express the monolayer parameter, $\kappa_{\mathrm{m}}$, in terms of the bilayer parameter, $\kappa_{\eta}$, we can use $\kappa_{\eta}=2 \kappa_{\mathrm{b}}$, and $\tilde{\kappa}$ can be expressed for a bilayer as $\tilde{\kappa}=\kappa_{\eta}\{1+$ $\left.48\left(h / 2 h_{t}\right)^{2}\right\} .{ }^{55}$ Considering the neutral surface as the interface between the hydrophilic headgroup and the hydrophobic tail $\left(h=h_{\mathrm{t}}\right),{ }^{56-60}$ we can redefine eq 11 to obtain the bending rigidity of a bilayer from $\mathrm{ULV}^{55}$

$$
\Gamma_{q}=0.0069 \frac{k_{\mathrm{B}} T}{\eta} \sqrt{\frac{k_{\mathrm{B}} T}{\kappa_{\eta}}}
$$

Equation 12 has been successfully used to calculate $\kappa_{\eta}$ for ULVs from NSE. ${ }^{41,46,55,61}$

For the special case of four monolayers (i.e., two bilayers or $N=2$ (neglecting any elastic effects from interbilayer water since they are predominantly viscous)), we can redefine $\kappa_{\eta}=$ $4 \kappa_{\mathrm{b}}$ and $\tilde{\kappa}=\kappa_{\eta}\left\{1+24\left(h / 2 h_{\mathrm{t}}\right)^{2}\right\}$, which results in

$$
\frac{\Gamma_{q}}{Q^{3}}=0.0094 \frac{k_{\mathrm{B}} T}{\eta} \sqrt{\frac{k_{\mathrm{B}} T}{\kappa_{\eta}}}
$$

In general, for $N$ layers (i.e., $2 N$ monolayers), we have $\kappa_{\eta}=$ $2 N \kappa_{\mathrm{b}}$ and $\tilde{\kappa}=\kappa_{\eta}(1+12 / N)$, which results in

$$
\frac{\Gamma_{q}}{Q^{3}}=0.025 \frac{k_{\mathrm{B}} T}{\eta} \sqrt{\frac{k_{\mathrm{B}} T}{\kappa_{\eta}(1+12 / N)}}
$$

According to this expression, the effective bending modulus for MLVs increases by a factor of $\frac{13 N}{(N+12)}$ for $N \geq 2$.

Additionally, we can analyze the mean-squared displacement (MSD) $\left\langle\Delta r(t)^{2}\right\rangle$ and the non-Gaussianity parameter $\alpha_{2}(t)=\frac{d}{d+2} \frac{\left\langle\Delta r(t)^{4}\right\rangle}{\left\langle\Delta r(t)^{2}\right\rangle^{2}}-1$ from the measured dynamic structure factor, $S(Q t)$, using a cumulant expansion given by $41,50,62,63$

$$
\frac{S(Q, t)}{S(Q)}=A \exp \left[-\frac{Q^{2}\left\langle\Delta r(t)^{2}\right\rangle}{6}+\frac{Q^{4} \alpha_{2}(t)}{72}\left\langle\Delta r(t)^{2}\right\rangle^{2}\right]
$$

Non-Gaussian parameter $\alpha_{2}$ is essentially defined as the quotient of the fourth $\left\langle\Delta r(t)^{4}\right\rangle$ and the second moment squared $\left\langle\Delta r(t)^{2}\right\rangle^{2}$, and $d=3$ is the dimension of space. ${ }^{41,63,64}$ Following eqs 10,12 , and 15 , we can express the membrane rigidity for ULVs as a function of Fourier time, given by ${ }^{50}$

$$
\frac{\kappa_{\eta}}{k_{\mathrm{B}} T}=\frac{t^{2}}{c(\eta, T)^{3}\left\langle\Delta r(t)^{2}\right\rangle^{3}}
$$


with $c(\eta, T)=\frac{1}{6}\left(\frac{\eta}{0.0069 k_{\mathrm{B}} T}\right)^{2 / 3}$. The $\mathrm{ZG}$ approximation $\left\langle\Delta r(t)^{2}\right\rangle \propto t^{2 / 3}$ and the bending rigidity as a function of time should yield $\kappa_{\eta} / k_{\mathrm{B}} T \propto t^{2} / t^{2}=$ constant. Any deviation from this constant behavior will reflect additional processes that are not considered in the ZG model. For MLVs, prefactor $c(\eta, T)$ needs to be modified following eq 14 .

\section{EXPERIMENTAL SECTION}

Sample Preparation. All chemicals and reagents were used as received. 1,2-Di(octadecenoyl)-sn-glycero-3-phosphocholine (DOPC) was purchased from Avanti Polar Lipids (Alabaster, AL, USA), and $\mathrm{D}_{2} \mathrm{O}$ was received from Sigma-Aldrich (St. Louis, MO, USA). We have used fully deuterated poly(ethylene oxide)-mono- $n$ octadecyl ether, $d$ - $\mathrm{C}_{18}-d$-PEO4, to reduce scattering contributions resulting from the contrast between solvent and polymer. The polymer was synthesized by living ring-opening, anionic polymerization of fully deuterated ethylene oxide, $d$-EO. The initiator was a mixture of deuterated 1-octacosanol and corresponding potassium 1octacosanolate. Exact polymerization conditions can be found in ref 37. The polymer was characterized by size-exclusion chromatography using a combination of the refractive index and an 18-angle light scattering detector (Optilab rEX and DAWN HELEOS-II, Wyatt) for absolute molecular weight characterization. For separation, three Agilent Plus Pore GPC columns with a continuous pore size distribution were used with a mixture of tetrahydrofuran, dimethylacetamide, and acetic acid as the eluent at a flux rate of 1 $\mathrm{mL} / \mathrm{min}$. The degree of polymerization of $d-\mathrm{PEO}$ is 92 , and the dispersity index is 1.03 . The PEO block has a number-average molecular weight of $M_{\mathrm{n}}=4420 \mathrm{~g} / \mathrm{mol}$.

DOPC vesicles were prepared by dissolving DOPC lipid powder in chloroform and removing the solvent using a rotary evaporator and drying further under vacuum overnight. The dried lipid was hydrated using $\mathrm{D}_{2} \mathrm{O}$, and the resultant solution was subjected to freeze-thaw cycling by alternatingly immersing the flask in the water at around 50 ${ }^{\circ} \mathrm{C}$ and placing it in a freezer at $-20{ }^{\circ} \mathrm{C}$ in 10 min intervals. Finally, the solution was extruded using a miniextruder (Avanti Polar Lipids, Alabaster, AL, USA) through a polycarbonate membrane with a pore diameter of $100 \mathrm{~nm}$ (33 passes) to obtain unilamellar vesicles (Figure S2). Vesicle solutions were mixed with $d-C_{18}-d-P E O 4$ solutions to obtain the desired external polymeric concentrations. This technique ensures the addition of the polymer from outside the vesicles. Measurements for each mixture were averaged starting $24 \mathrm{~h}$ after sample preparation. All experiments were conducted at ambient temperature.

DLS. Dynamic light scattering (DLS) measurements were performed using a Malvern Zetasizer Nano ZS ( $\mathrm{He}-\mathrm{Ne}$ laser wavelength, $\lambda=633 \mathrm{~nm}$ at $30 \mathrm{~mW}$ laser power, at a backscattering setup angle of $\theta=173^{\circ}$. The hydrodynamic radius, $R_{\mathrm{h}}$, of the liposomes in each $d-\mathrm{C}_{18}-d$-PEO4 concentration was calculated from the translational diffusion coefficient, $D_{\mathrm{t}}$ using the Stokes-Einstein equation, $R_{\mathrm{h}}=k_{\mathrm{B}} T /\left(6 \pi \eta_{0} D_{\mathrm{t}}\right)$, with the Boltzmann constant, $k_{\mathrm{B}}$, the temperature, $T$, and the viscosity of the solvent, $\eta_{0}$. DLS measurements were performed in triplicate for each mixture and averaged. In Figure S3 and Table S1, the results from DLS are reported for $5 \mathrm{wt} \%$ DOPC along with three different concentrations of $d-\mathrm{C}_{18}-d-\mathrm{PEO} 4$ polymer added to the $5 \mathrm{wt} \%$ DOPC liposome solution. To calculate the corresponding $R_{\mathrm{h}}$, we have used the viscosity of $5 \mathrm{wt} \% \mathrm{DOPC}$ as the solvent viscosity, $\eta_{0}=(1.6 \pm 0.03) \times 10^{-3} \mathrm{~Pa} \cdot \mathrm{s}$ (cf. Supporting Information).

Cryo-TEM. Cryogenic-transmission electron microscopy (cryoTEM) images were recorded on a Tecnai G2 F30 operated at $150 \mathrm{kV}$. A volume of $10 \mu \mathrm{L}$ of the DOPC vesicles or DOPC polymer mixture sample was applied to a 200-mesh lacey carbon grid mounted on the plunging station of an FEI Vitrobot, and excess liquid was blotted for $2 \mathrm{~s}$ by the filter paper attached to the arms of the Vitrobot. The carbon grids with the attached thin films with aqueous solutions of vesicles were plunged into liquid ethane and transferred to a single tilt cryo-specimen holder for imaging. By quick plunging into liquid ethane, the vesicles are preserved in their hydrated state present at room temperature. Cryo-TEM images were obtained in the bright field setting. The DOPC vesicle concentration was maintained at 0.25 wt $\%$ or below to suit sample preparation and facilitate visualization. For vesicle-polymer mixtures, cryo-TEM images were taken for 0.25 wt $\% \mathrm{DOPC}+1$ wt $\% \mathrm{C}_{18}$-PEO4, and another set of images were taken with 0.125 wt $\%$ DOPC and 0.03 wt $\% \mathrm{C}_{18}$-PEO4 by maintaining the DOPC/polymer weight ratio of $\sim 5: 1$ comparable to the ratio used for other techniques. All size analyses on cryo-TEM images were carried out using ImageJ software.

SANS. Small-angle neutron scattering (SANS) experiments were conducted at the NG 7 SANS instrument of the NIST Center for Neutron Research (NCNR) at the National Institute of Standards and Technology (NIST). ${ }^{65}$ The sample-to-detector distances, $d$, were set to 1,4 , and $13 \mathrm{~m}$ at a neutron wavelength of $\lambda=6 \AA$. Another configuration with lenses at $d=15.3 \mathrm{~m}$ and $\lambda=8 \AA$ was used to access low $Q$ values. ${ }^{66}$ This combination covers a $Q$ range from 0.001 to 0.6 $\AA^{-1}$, where $Q=4 \pi \sin (\theta / 2) / \lambda$ with a scattering angle of $\theta$. A wavelength resolution of $\Delta \lambda / \lambda=14 \%$ was used. All data reduction to intensity, $I(Q)$, vs momentum transfer, $Q=|\overrightarrow{\mathrm{Q}}|$, was carried out following the standard procedures that are implemented in the NCNR macros to the Igor software package. ${ }^{67}$ The intensity values were scaled to absolute units $\left(\mathrm{cm}^{-1}\right)$ using a direct beam. $\mathrm{D}_{2} \mathrm{O}$ as the solvent and empty cells were measured separately. The polymer solution composed of $1 \mathrm{wt} \% d-\mathrm{C}_{18}-d$-PEO4 polymer in $\mathrm{D}_{2} \mathrm{O}$ was measured separately and subtracted as the background.

SAXS. Small-angle X-ray scattering (SAXS) experiments were conducted at the LIX beamline at National Synchrotron Light Source II, Brookhaven National Laboratory, and at the Bio-SAXS beamline at the Stanford Linear Accelerator Center (SLAC) facility. The samples were measured in a flow cell to avoid damage due to the intense photon beam, with an acquisition time of $1 \mathrm{~s}$. The recorded intensities were corrected for dark current, empty cell, and solvent (buffer) using standard procedures. ${ }^{68,69}$ The polymer solution composed of 1 wt \% $d$ - $\mathrm{C}_{18^{-}} d$-PEO4 polymer in $\mathrm{D}_{2} \mathrm{O}$ was measured separately and subtracted as the background.

NSE. Neutron spin echo (NSE) spectroscopy has been used to examine the effects of membrane dynamics simultaneously over broad length and time scales. We obtained NSE data at BL-15 at the Spallation Neutron Source of the Oak Ridge National Laboratory, Oak Ridge, TN. ${ }^{70}$ We used Hellma quartz cells with a $2 \mathrm{~mm}$ sample thickness. The lipid concentration was always 5\%. Measurements were conducted using a wavelength of $8 \AA$. The BL15 ECHODET software package was used for data reduction. $\mathrm{D}_{2} \mathrm{O}$ and solutions of 1 wt $\% d$ - $\mathrm{C}_{18}-d$-PEO4 and $\mathrm{D}_{2} \mathrm{O}$ were measured separately and used for background subtraction.

\section{RESULTS AND DISCUSSION}

We first evaluated the size, shape, and morphology of the vesicle from DLS, cryo-TEM, and SANS, whereas the lipid bilayer structure is determined from SAXS. This helps us to examine the number of bilayers in the vesicle, the amount of polymer interacting with the lipid bilayer, and the effect of polymer deposition on the outer layer of the vesicle. SAXS and cryo-TEM results are used to quantify the effect of polymerinduced perturbation of the liposome structure. Next, we measure the dynamics of the phospholipid membrane by NSE to determine the effect of change in the structure on the membrane rigidity. All of these results are brought together to comprehend the mechanism of polymer-induced transformation of the vesicle structure and dynamics.

Structure and Morphology. The vesicle size is determined by analyzing the intensity autocorrelation function, $g^{2}(Q t)$, measured by DLS using a single-exponential decay:

$$
g^{2}(Q, t)=A \exp \left(-2 D_{t} Q^{2} t\right)
$$



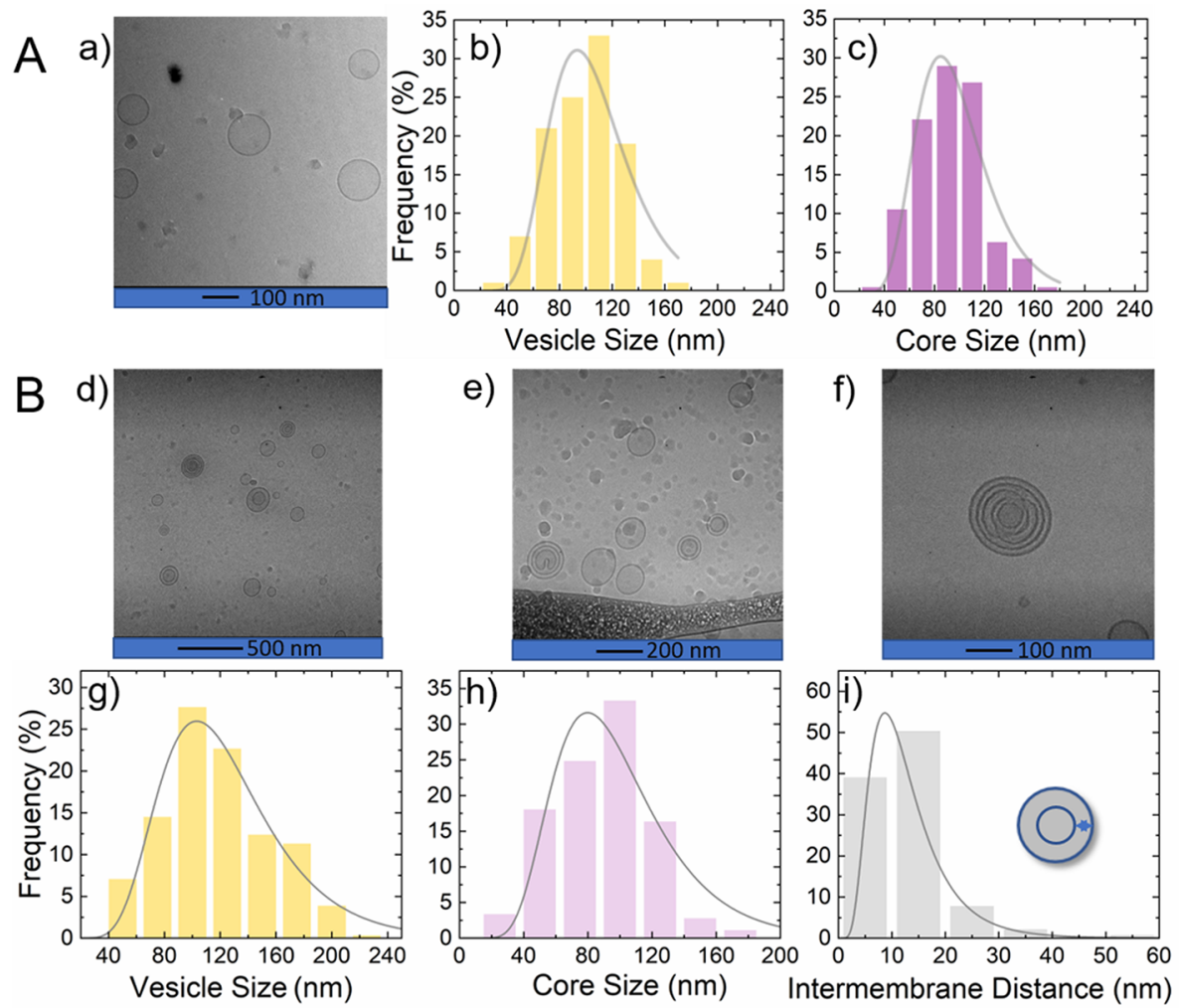

Figure 1. (A) Cryo-TEM analysis of 0.25 wt \% pure DOPC vesicles. (a) Cryo-TEM image for pure DOPC vesicles, (b) log-normal distribution of the corresponding size distribution, and (c) the inner water core size. (B) Cryo-TEM analysis for $0.125 \mathrm{wt} \%$ DOPC vesicles with 0.03 wt $\% d$ - $\mathrm{C}_{18^{-}}$ $d$-PEO4 polymer (lipid/polymer w/w ratio 5:1). (d-f) Cryo-TEM images of the vesicle-polymer mixture, $(\mathrm{g})$ corresponding log-normal size distribution of the vesicle size, $(h)$ the innermost water core size, and (i) the interbilayer water thickness.

The corresponding diffusion coefficients and hydrodynamic radii are summarized in Table $\mathrm{S} 1$ for different concentrations of $d$ - $\mathrm{C}_{18}$ - $d$-PEO4 polymers in $5 \mathrm{wt} \%$ DOPC. The corresponding autocorrelation functions and diffusion distributions are also reported in the Supporting Information (SI). While the 1 wt $\% d$ - $\mathrm{C}_{18}$-PEO4 samples have a larger hydrodynamic radius $(68 \pm 2 \mathrm{~nm})$, lower concentrations of $0,0.25$, and $0.5 \mathrm{wt} \%$ have little to no influence on the size $(60,62$, and $61 \mathrm{~nm}$ with $\pm 2 \mathrm{~nm}$ error). The width of the log-normal distribution is constant within the experimental accuracy.

The morphology of the vesicles was observed using cryoTEM. Figures 1 and 2 summarize cryo-TEM images and analyses for DOPC vesicles and DOPC vesicles in the presence of polymer. Figure 1(A-a) illustrates the cryo-TEM for $0.25 \mathrm{wt}$ $\%$ pure DOPC vesicles in $\mathrm{D}_{2} \mathrm{O}$. Figure $1(\mathrm{~A}-\mathrm{b}, \mathrm{c})$ shows the respective vesicle size and water core size analysis obtained using log-normal fits. The images and size analysis of pure DOPC vesicles presented in Figure $1(\mathrm{~A}-\mathrm{a}-\mathrm{c})$ are based on our earlier data. ${ }^{41}$ Because cryo-TEM experiments require low concentrations for sample preparation, 5 wt \% DOPC vesicles in the presence of 1 wt $\% d-\mathrm{C}_{18}-d$-PEO4 cannot be observed for direct comparison with SAXS and SANS. However, we measured a 0.125 wt $\%$ DOPC and 0.03 wt $\% d-C_{18}-d$-PEO4 mixture maintaining the ratio between lipids and polymers similar to that in SAXS and SANS experiments, $\sim 5: 1(\mathrm{w} / \mathrm{w})$. Figure 1(B-d-f) shows cryo-TEM images of $0.125 \mathrm{wt} \%$ DOPC vesicles dispersed in 0.03 wt $\% d-C_{18}-d$-PEO4 polymer solution. These indicate the presence of a highly polydisperse mixture of unilamellar and multilamellar vesicles (MLV). The data analysis yields a log-normal size distribution for the vesicle
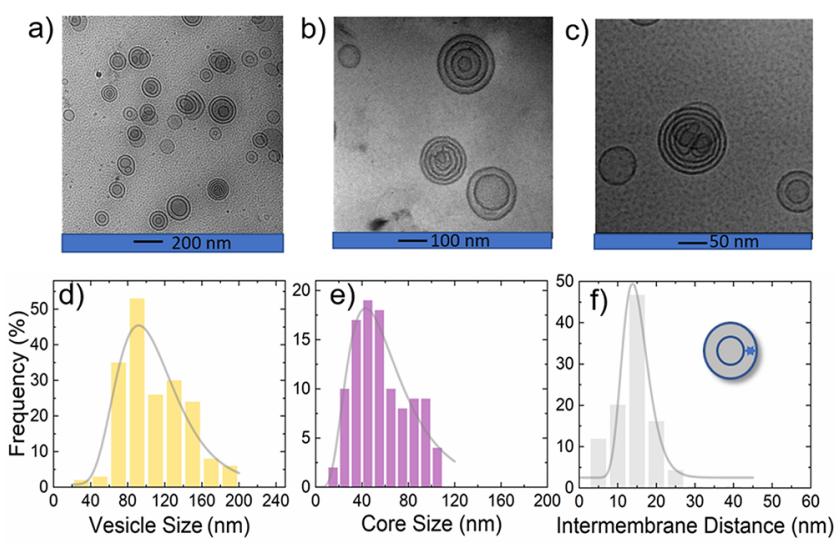

Figure 2. Cryo-TEM images and analyses for $0.25 \mathrm{wt} \% \mathrm{DOPC}$ with 1 wt $\% d$ - $\mathrm{C}_{18}-d$-PEO4 in $\mathrm{D}_{2} \mathrm{O}$. (a-c) Cryo-TEM images at different magnifications and $(\mathrm{d}-\mathrm{f})$ size analyses. The solid lines represent lognormal size distributions of $(d)$ vesicle size, $(e)$ core size, and $(\mathrm{f})$ intermembrane distances, respectively. The data are tabulated in Table 1.

size, innermost water core size, and interbilayer water thickness as shown in Figure $1 \mathrm{~B}-\mathrm{g}-\mathrm{i}$, respectively.

Because of the low count of vesicles in the cryo-TEM sample maintained at a 5:1 lipid weight ratio, we further explored 0.25 wt $\%$ DOPC with 1 wt $\% d-C_{18}-d$-PEO4 polymer data summarized in Figure 2 where the $d-\mathrm{C}_{18}-d$-PEO4 concentration is similar to that determined from the other techniques described. In both cases, multilamellar vesicle formation and increasing vesicle size were observed. 
Table 1. Structural Parameters from Cryo-TEM and SANS ${ }^{a}$

\begin{tabular}{|c|c|c|c|c|}
\hline \multirow[b]{2}{*}{ samples } & \multicolumn{2}{|c|}{ cryo-TEM } & \multicolumn{2}{|c|}{ SANS } \\
\hline & 0 wt $\% d-\mathrm{C}_{18}-d-\mathrm{PEO} 4$ & 1 wt $\% d-\mathrm{C}_{18}$-PEO4 & 0 wt $\% d-\mathrm{C}_{18}-\mathrm{PEO} 4$ & 1 wt $\% d-\mathrm{C}_{18}-\mathrm{PEO} 4$ \\
\hline$N$ & 1 & $\sim 3$ & 1 & 2 \\
\hline$R_{\mathrm{p}}(\mathrm{nm})$ & $51 \pm 3$ & $52 \pm 4$ & $54 \pm 2$ & $59 \pm 2$ \\
\hline$R_{\mathrm{c}}(\mathrm{nm})$ & $47 \pm 2$ & $28 \pm 1$ & $51 \pm 2$ & $37 \pm 2$ \\
\hline$t_{\mathrm{w}}(\mathrm{nm})$ & $\mathrm{NA}^{c}$ & $15 \pm 1$ & $\mathrm{NA}^{c}$ & $15 \pm 1$ \\
\hline$T_{\mathrm{s}}(\mathrm{nm})$ & $\mathrm{NA}^{b}$ & $\mathrm{NA}^{b}$ & $3.6 \pm 0.1$ & $3.4 \pm 0.2$ \\
\hline$R_{\mathrm{p}}$ polydispersity (\%) & $30 \pm 7$ & $34 \pm 9$ & $30 \pm 2$ & $40 \pm 2$ \\
\hline$R_{\mathrm{c}}$ polydispersity (\%) & $30 \pm 6$ & $52 \pm 5$ & $30 \pm 2$ & $40 \pm 2$ \\
\hline$t_{\mathrm{w}}$ polydispersity $(\%)$ & $\mathrm{NA}^{c}$ & $23 \pm 3$ & $\mathrm{NA}^{c}$ & $41 \pm 4$ \\
\hline
\end{tabular}

${ }^{a}$ Number of layers $(N)$, outer perimeter radius of the vesicle $\left(R_{\mathrm{p}}\right)$, water core radius $\left(R_{\mathrm{c}}\right)$, intermembrane water thickness $\left(t_{\mathrm{w}}\right)$, bilayer thickness $\left(t_{\mathrm{s}}\right)$, and corresponding size polydispersity. ${ }^{b}$ Not visible in TEM images. ${ }^{c}$ Not applicable to ULVs.

The corresponding vesicle perimeter radius yields $R_{\mathrm{p}, \mathrm{TEM}}=$ $52 \pm 4 \mathrm{~nm}$ with $34 \pm 9 \%$ polydispersity. The water core size analysis yields a core radius of $R_{\mathrm{c}, \text { TEM }}=28 \pm 1 \mathrm{~nm}$ with $52 \pm$ $5 \%$ polydispersity. Figure $2(\mathrm{f})$ shows the average interbilayer water thickness of $t_{\mathrm{w}, \mathrm{TEM}}=14.6 \pm 0.5 \mathrm{~nm}$ and its polydispersity of $23 \pm 3 \%$. While the addition of the polymer leaves the perimeter diameter slightly increased compared to the pure DOPC vesicles with $R_{\mathrm{p}, \mathrm{TEM}}=51 \pm 3 \mathrm{~nm}$ and $30 \pm 7 \%$ polydispersity, the core size is substantially changed compared to the pure DOPC vesicles with $R_{c, T E M}=47 \pm 1 \mathrm{~nm}$ and $30 \pm$ $6 \%$ polydispersity. This significant reduction in the size of the water core in the presence of $d-\mathrm{C}_{18}-d$-PEO4 comes with a substantial increase in polydispersity. For a more direct comparison, Table 1 summarizes the values. We obtained $R_{\mathrm{p}, \mathrm{TEM}}, R_{\mathrm{c}, \mathrm{TEM}}$, and $t_{\mathrm{w}, \mathrm{TEM}}$ values by counting as many as 187 , 106 , and about 278 distances, respectively, including different orientations. Compared to scattering experiments, this number is still low; therefore, we used scattering experiments for improved statistical representation.

Figure 3 presents SANS data of 5 wt \% DOPC in $\mathrm{D}_{2} \mathrm{O}$ and 5 wt $\%$ DOPC mixed with 1 wt $\% d-C_{18}-d-P E O 4$. The solid red

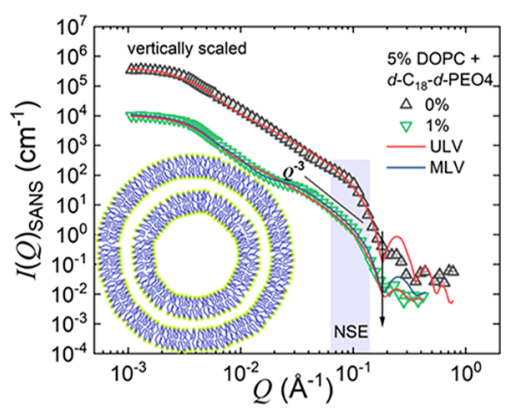

Figure 3. SANS scattering data for pure $5 \mathrm{wt} \%$ DOPC and DOPC mixed with 1 wt $\% d$ - $\mathrm{C}_{18}-d$-PEO4 polymer dispersed in $\mathrm{D}_{2} \mathrm{O}$. The solid lines represent the ULV $(N=1)$ and $\operatorname{MLV}(N=2)$ model described in eqs 1,2, and 4 . The shaded region depicts the $Q$ range over which NSE experiments are performed. The inset depicts the schematic illustration of the $\operatorname{MLV}(N=2)$ model. The $0 \mathrm{wt} \%$ data is vertically unscaled, and the DOPC mixed with $1 \mathrm{wt} \% d-\mathrm{C}_{18}-\mathrm{d}$-PEO4 data is vertically scaled, multiplied by a factor $c(c=30)$, for better visualization

lines represent the data modeling using the vesicle form factor as described in eqs 1,2 , and 4 . For both pure vesicles and vesicles with 1 wt $\% d$ - $C_{18}$ - $d$-PEO4 polymer samples, we illustrate data modeling using a ULV form factor $(N=1)$. As shown in Figure 3, although we obtain a satisfactory description for the pure DOPC, we cannot explain the vesicles with 1 wt $\% d-\mathrm{C}_{18}-d$-PEO4 data using a ULV model. We observe a $Q^{-3}$ power-law dependence over the $Q$ range of 0.02 to $0.08 \AA^{-1}$, which might be due to scattering from a highly folded and convoluted surface arising from the adsorption of the polymer on the vesicle surface. ${ }^{71,72}$ Such surfaces are also visible in the cryo-TEM images (Figures $1 \mathrm{~B}(\mathrm{~d}-\mathrm{f}$ ) and $2(a, b, c))$ along with the formation of MLVs. In this case, the presence of the polymer facilitates MLV formation. We modeled the SANS data for the 1 wt $\% d-\mathrm{C}_{18}-d$-PEO4DOPC sample using the MLV model with the water core encapsulated by $N=2$ lipid shells. The inset figure represents the SANS model used in data modeling. The parameters obtained from the TEM analysis, like the core radius, $R_{\mathcal{c}}$ and the interbilayer water thickness, $t_{\mathrm{w}}$, were used as an initial estimation for fitting to obtain the outer perimeter radius $R_{\mathrm{p}}$ as presented in Table 1 . The data fitting was done with a maximum of two or three free parameters at a time. We varied each of the parameters systematically again and again in a loop until a negligible change in the fitted curve and residual had been achieved. We covered all of the parameters reported in Table 1, where the error bars represent the maximum deviation for each parameter over which our model can describe the data. Therefore, they represent the uncertainties in the parameters. The plots of residuals for the fits are included in the SI.

As suggested by DLS and cryo-TEM experiments, we used a log-normal distribution for the polydispersity. From SANS, we obtain a $9 \%$ increase in the size of the DOPC vesicles, $R_{\text {SANS }}=$ $53.6 \pm 0.2 \mathrm{~nm}\left(0 \mathrm{wt} \% d-\mathrm{C}_{18}-d-\mathrm{PEO} 4\right)$ to $57.9 \pm 0.5 \mathrm{~nm}(1 \mathrm{wt}$ $\% d-\bar{C}_{18}-d$-PEO4). In the presence of 1 wt $\% d-C_{18}-d$-PEO4 polymer, along with the formation of MLVs we observe an $\sim 5 \%$ reduction in the bilayer thickness and find an interbilayer water layer of $t_{\mathrm{w}} \approx 14 \mathrm{~nm}$. Both SANS and cryo-TEM yield a large polydispersity of $t_{\mathrm{w}}$.

An estimate of the amount of polymer interacting with phospholipid vesicles was obtained by additional SANS experiments. Figure $S 9$ in the SI illustrates SANS data for (i) 0.25 wt $\%$ DOPC in $\mathrm{D}_{2} \mathrm{O}$, (ii) 5 wt $\%$ of $h-\mathrm{C}_{18}-h$-PEO4 in $\mathrm{D}_{2} \mathrm{O}$, and (iii) 0.25 wt $\%$ DOPC with 5 wt $\% h-C_{18}-h$-PEO4 in $\mathrm{D}_{2} \mathrm{O}$ (mixture). The black line represents the weighted sum of intensities of samples (i) and (ii). While these results indicate that the blend of $h-\mathrm{C}_{18}-h-\mathrm{PEO} 4$ and DOPC has characteristic features of the micellar structure factor and the unperturbed DOPC liposomes, deviations indicate interactions between polymer and liposome. The data in Figure S9 is particularly useful because the weighted addition tells us that the $5 \mathrm{wt} \%$ polymer fraction that forms micelles is reduced to $4.7 \mathrm{wt} \%$. 
Since the critical micellar concentration (CMC) of $h-\mathrm{C}_{18}-h$ PEO4 is 0.01 wt $\%,{ }^{73}$ we calculated that 0.3 wt $\%$ of the polymer interacts with $0.25 \mathrm{wt} \%$ of the DOPC vesicles. This number accounts for $6 \%$ of the added $h$ - $\mathrm{C}_{18}$ - $h$-PEO4. It is very likely that the system of micelles and liposomes dynamically exchanges unimers. In this way, $94 \%$ of the unimers form micelles, and $6 \%$ refers to the number of molecules that are dynamically exchanging. Hence, this $6 \%$ refers to the maximum amount, while the average amount of polymer inserted in the liposome can be significantly lower. This information is used to calculate the lipid/polymer ratio in the lipid layer and contrast with conditions arising later.

Figure 4 compares SAXS on 5 wt \% DOPC vesicles in $\mathrm{D}_{2} \mathrm{O}$ with 5 wt $\%$ DOPC and the 1 wt $\% d-\mathrm{C}_{18}-d-\mathrm{PEO} 4$ mixture.

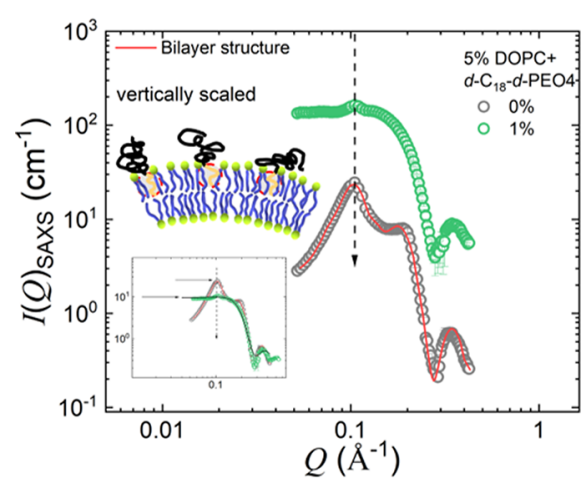

Figure 4. SAXS diffraction data for pure DOPC and DOPC mixed with a 1 wt $\% d-\mathrm{C}_{18}-d$-PEO4 polymer dispersed in $\mathrm{D}_{2} \mathrm{O}$. The solid red line is the data modeled using the MLV form factor. The data is vertically scaled. The inset depicts the unscaled data. The schematic illustration of the bilayer structure with the polymers wedged in is depicted. The $1 \mathrm{wt} \%$ data is vertically scaled by a factor of $c=15$ for proper visualization. Further SAXS data analysis is in the SI.

Represented by the solid red line, the aqueous solution with only DOPC is modeled by the Caille structure factor of multilamellar vesicles (eqs 5, 6, and 7). We find $N=2$ layers with a lamellar repeat distance $d=6.6 \pm 0.4 \mathrm{~nm}$ and a head-tohead bilayer thickness $\delta_{\mathrm{HH}}=4.5 \mathrm{~nm}$. We point to the observation of $N=2$ (SAXS) and 1 (SANS) layers, though we use the same sample in SAXS and SANS. There is no indication that the sample has been affected by any radiation damage as we used a flow cell. However, the experiments of Courbin et al. ${ }^{74}$ suggest the formation of multilamellar vesicles by shear. This is certainly not any proof of our observation, but it could point to more detailed investigations of flow-induced effects. A recent paper by Heberle et al. discusses another possibility where a small percentage of MLVs may be present even without being subjected to flow. They have shown that even after extrusion through polycarbonate membranes to synthesize unilamellar vesicles, a small fraction of MLVs may be present, and this will give rise to a SAXS profile that is intermediate between MLVs and ULVs even with a small percentage of MLVs. ${ }^{75}$ Therefore, this could be another reason for the observation of $N=2$ (SAXS) and 1 (SANS) layers for pure DOPC vesicles.

In the presence of $d-\mathrm{C}_{18}-d$-PEO4 polymers, the most obvious observation is the substantially decreasing intensity of the peak at $Q \approx 0.1 \AA^{-1}$ (vertical dashed arrow). The inset shows that the second peak (or shoulder) roughly at $Q \approx 0.2$ $\AA^{-1}$ disappears as well. A separation of the intensity in the product of the structure and form factor (Figure S10 in the SI) suggests that the observed changes arise from a perturbation of the lamellar repeat distance, $d$, which indicates a more heterogeneous system, supporting the independent information by cryo-TEM.

We used SANS results summarized in Table 1 to calculate the number of lipids and the average cross-sectional or lateral space that lipid molecules assume in distinct leaflets. The total number of lipids (per liposome) $N$ is given by, $N=\sum_{i} N_{i}$, with $N_{i}$ being the number of lipids in each leaflet $i$. Our system is bilamellar, thus amounting to four leaflets and hence $N=N_{1}+$ $N_{2}+N_{3}+N_{4}$.

If we assume that the cross-sectional area of one lipid, $\alpha$, is known, then $N_{i}=\frac{A_{i}}{a}$, with $A_{i}$ referring to the surface area of one leaflet. The liposome surface area is $A_{i}=\pi R_{i}^{2}$, with $R_{i}$ being the radius, which is known from SANS. To estimate the number of lipids in each leaflet, we assume that the area per headgroup, $\alpha$, is independent of the curvature of the leaflet. Because the radii are only slightly different, this approximation works very well, as indicated by the results. In the main text, we report the core radius, $R_{\mathcal{C}}$ the thickness of the bilayer, $\delta_{\mathrm{HH}}$, and the thickness of the water layer, $t_{\mathrm{w}}$ (cf. Table 1 ). In this way, we identify $R_{1}=R_{\mathrm{c}}, R_{2}=R_{\mathrm{c}}+\delta_{\mathrm{HH}}, R_{3}=R_{\mathrm{c}}+\delta_{\mathrm{HH}}+t_{\mathrm{w}}$, and $R_{4}=R_{\mathrm{c}}$ $+2 \delta_{\mathrm{HH}}+t_{\mathrm{w}}$. Using $N_{i}=\frac{R_{i}}{a}$, we arrive at $N_{1} a=4 \pi R_{\mathrm{c}}^{2}, N_{2} a=$ $4 \pi\left(R_{\mathrm{c}}+\delta_{\mathrm{HH}}\right)^{2}, N_{3} a=4 \pi\left(R_{\mathrm{c}}+\delta_{\mathrm{HH}}+t_{\mathrm{w}}\right)^{2}$, and $N_{4} a=4 \pi\left(R_{\mathrm{c}}+\right.$ $\left.2 \delta_{\mathrm{HH}}+t_{\mathrm{w}}\right)^{2}$.

As

$$
\begin{aligned}
& N a=\left(N_{1}+N_{2}+N_{3}+N_{4}\right) a \\
& =4 \pi\left[R_{\mathrm{c}}^{2}+\left(R_{\mathrm{c}}+\delta_{\mathrm{HH}}\right)^{2}+\left(R_{\mathrm{c}}+\delta_{\mathrm{HH}}+t_{\mathrm{w}}\right)^{2}\right. \\
& \left.\quad+\left(R_{\mathrm{c}}+2 \delta_{\mathrm{HH}}+t_{\mathrm{w}}\right)^{2}\right]
\end{aligned}
$$

we obtain $a=\frac{4 \pi\left[R_{\mathrm{c}}{ }^{2}+\left(R_{\mathrm{c}}+\delta_{\mathrm{HH}}\right)^{2}+\left(R_{\mathrm{c}}+\delta_{\mathrm{HH}}+t_{\mathrm{w}}\right)^{2}+\left(R_{\mathrm{c}}+2 \delta_{\mathrm{HH}}+t_{\mathrm{w}}\right)^{2}\right]}{N}$

$$
=0.73 \pm 0.02 \mathrm{~nm}^{2}
$$

and $N_{1}=23000 \pm 1200, N_{2}=27900 \pm 1500, N_{3}=52500 \pm$ 3900 , and $N_{4}=59000 \pm 4400$ with $N=163100 \pm 11200$ as estimates of the number of lipids in each leaflet and one vesicle.

If we assume a lipid headgroup to be circular, then $a=\pi r^{2}$ yields the radius $r=0.48 \pm 0.07 \mathrm{~nm}$ or diameter $d=0.96 \pm$ $0.15 \mathrm{~nm}$ a lipid occupies. It should be noted that a lipid head surface area of $0.69 \pm 0.02 \mathrm{~nm}^{2}$ was obtained in our previous work, ${ }^{41}$ yielding $r=0.47 \pm 0.01 \mathrm{~nm}$. From the SAXS study, an area of $0.72 \pm 0.005 \mathrm{~nm}^{2}$ was reported, ${ }^{76}$ yielding $r=0.47 \pm$ $0.01 \mathrm{~nm}$. These results agree very well. Furthermore, in neutron spin echo or quasi-elastic neutron scattering experiments, the lipid tail motion has been analyzed by assuming that the lipid tails relax in an environment that is described by a cylindrical potential with a cylinder radius of approximately $0.43 \mathrm{~nm} .{ }^{50}$ This number is very similar to the radius obtained by the entirely independent estimate via the number of lipids per leaflet. Therefore, all experiments seem to be in favor of lipids that occupy on average a cylindrical area with the diameter being roughly $1 \mathrm{~nm}$.

Using the above information and SANS data on DOPC and $h$ - $\mathrm{C}_{18}-h$-PEO4, the relative fraction of polymer in the mixture of 5 wt $\%$ DOPC vesicles with 1 wt $\% d-C_{18}-d-P E O 4$ is attempted. As explained in the SI $(N=2$, page S7), considering that all polymers interact with only the outermost leaflet, out of a total of $5 \mathrm{wt} \%$ DOPC lipids in $1 \mathrm{~mL}=6.36 \times$ 

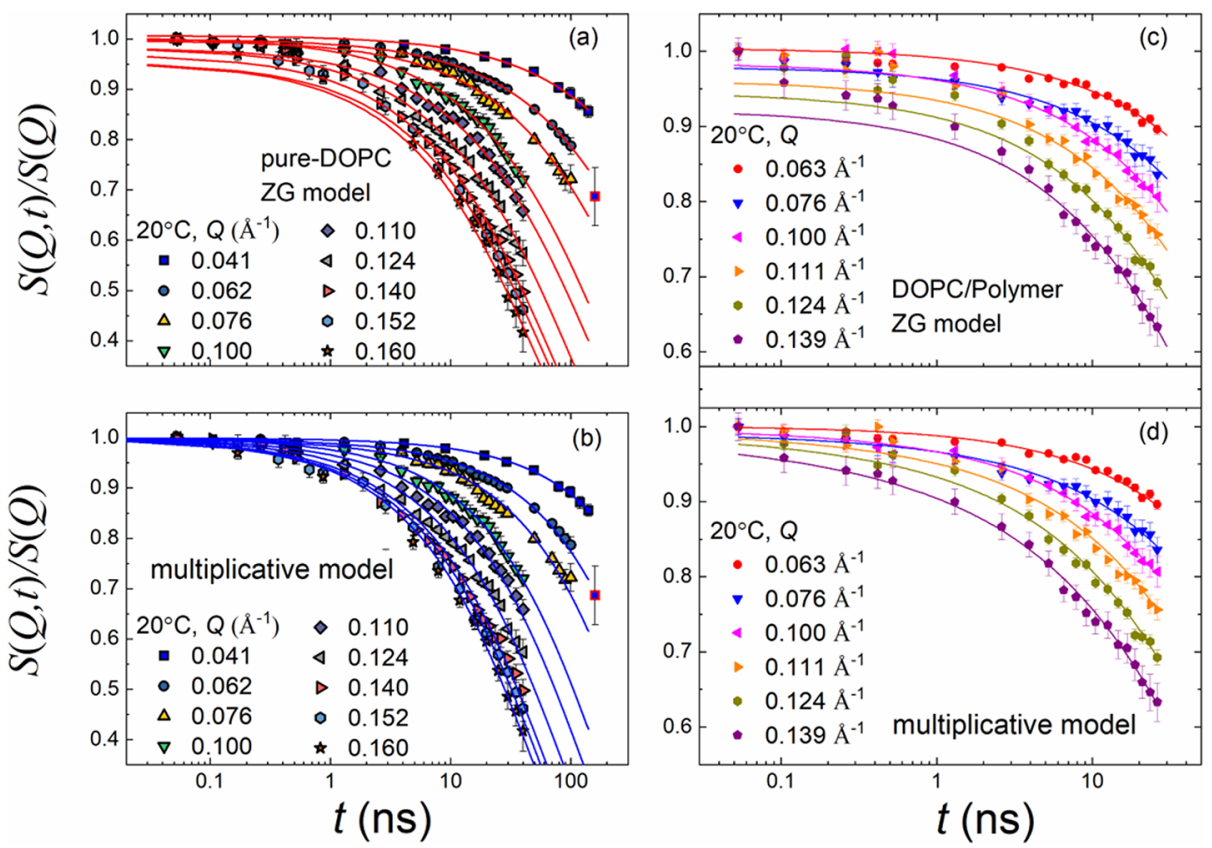

Figure 5. Lin-log representations of the normalized dynamic structure factor, $S(Q, t) / S(Q)$, as a function of Fourier time, $t$, for different $Q$ 's, pure DOPC vesicles $(\mathrm{a}, \mathrm{b})$, and DOPC mixed with $1 \%$ mass fraction of $d-\mathrm{C}_{18}-d$-PEO4 polymer dispersed in $\mathrm{D}_{2} \mathrm{O}$ at $20{ }^{\circ} \mathrm{C}$ (c, d). The same data sets are analyzed by fits using the Zilman Granek model (eq 10) and the multiplicative model described in eq 9 that includes diffusion and confined motion. The error bars represent one standard deviation. A lin-lin plot for DOPC mixed with polymer is presented in the SI. The data for pure DOPC were reproduced from our previous studies. ${ }^{41,50}$

Table 2. Membrane Rigidity $\kappa_{\eta}$ Obtained for a $1 \mathrm{wt} \% d-\mathrm{C}_{18^{-}} d$-PEO4-DOPC Sample Using Different Models for $D_{\mathrm{t}}=0$ and $\eta=$ $\eta_{\mathrm{D}_{2} \mathrm{O}}{ }^{a}$

\begin{tabular}{|c|c|c|c|c|c|c|}
\hline \multicolumn{7}{|c|}{$\kappa_{\eta} / k_{\mathrm{B}} \mathrm{T}$} \\
\hline parameters & concentration $d-\mathrm{C}_{18}$-PEO4, wt \% & $N$ & ZG analysis (full-time range) (eq 10) & ZG analysis $(t>5 \mathrm{~ns})$ & multiplicative model (eq 9) & MSD analysis \\
\hline$D_{\mathrm{t}}=0$ & 0 & 1 & $26 \pm 1$ & $20 \pm 2$ & $21 \pm 2$ & $18 \pm 2$ \\
\hline \multirow[t]{2}{*}{$\eta=\eta_{\mathrm{D}_{2} \mathrm{O}}$} & 1 & 2 & $26 \pm 5$ & $28 \pm 5$ & $29 \pm 5$ & $30 \pm 3$ \\
\hline & 1 & 1 & $13+3$ & $14+4$ & $15+3$ & $15 \pm 2$ \\
\hline
\end{tabular}

${ }^{a}$ We used the results of the $0 \mathrm{wt} \% d$-C ${ }_{18} d$-PEO4-DOPC sample from our previous study. ${ }^{41,50,61}$ For comparison with pure DOPC, we have included $\kappa_{\eta}$ for $N=2$ and 1 layers.

$10^{-5} \mathrm{~mol} \times 6.02 \times 10^{23} \mathrm{~mol}^{-1}=3.83 \times 10^{19}$ lipids, the number of lipids in the total outer leaflets per milliliter was $1.39 \times 10^{19}$. Since there are $2.34 \times 10^{14}$ vesicles per $\mathrm{mL}$, we obtain $1.24 \times$ $10^{18} d-\mathrm{C}_{18}$ - $d$-PEO4 per $\mathrm{mL}$ inserted into the outer layer.

As shown on SI page S7, Figure S9, if we compare fully hydrogenated samples, the molar ratio for $0.25 \mathrm{wt} \%$ DOPC to 0.3 wt $\% h-C_{18}-h$-PEO4 is $2: 1$, which interacts with the outer leaflet of the outer lipid bilayer. We argued that there might be a dynamic exchange, hence $0.3 \mathrm{wt} \%$ interacts with the bilayer, while 4.7 wt \% of the polymers form micelles and stay in the aqueous phase. The molar ratio in the case of $5 \mathrm{wt} \%$ DOPC to 1 wt $\% h-C_{18}-h$-PEO4 is $11: 1$. This suggests that all of the $1 \mathrm{wt}$ $\%$ polymers interact with the outer leaflet of the vesicle because the saturation level of $5.6 \mathrm{wt} \%$ is not reached when excess polymers are present to form micelles. Thus, we have a lipid fraction of 0.91 or a polymer fraction of 0.08 if we consider only the outer leaflet, and we would assume that all polymers are in this outer leaflet. If the polymer interacts equally with all four leaflets, then this number would be 4 times lower and would be less than 0.02 . Whenever we assume that all of the polymer inserts in the vesicles, the concentration range for the outer leaflet ranges from 0.02 to 0.08 . The polymer concentration is very low compared to the lipid concentration.
Therefore, it is in favor of approximating the cross-sectional area, $a$, of a lipid by a constant value.

Dynamics. Figure 5(c) illustrates the dynamic structure factor, $S(Q, t)$, measured by NSE for the blend of the aqueous $\left(\mathrm{D}_{2} \mathrm{O}\right)$ solutions with 1 wt $\% d-\mathrm{C}_{18}-d-\mathrm{PEO} 4$ and $5 \mathrm{wt} \%$ DOPC, covering a $Q$ range from 0.063 to $0.139 \AA^{-1}$. The solid lines represent the description by the ZG model (eq 10). The logarithmic time axis accentuates deviations at low Fourier times and indicates processes beyond membrane undulations. The membrane rigidities, $\kappa_{\eta} /\left(k_{\mathrm{B}} T\right)$, as calculated from eq 13 (for $N=2$ layers) are listed in Table 2. For a better comparison, we have included the results of pure DOPC $(N=$ 1) from our previous studies in Figure $5(\mathrm{a}, \mathrm{b}) .^{41,50}$

The observation of three different processes in neat protiated DOPC - translational diffusion of the vesicle, ZG membrane undulation, and confined tail motion-suggests using the multiplicative model (eq 9). Figure 5(d) shows better agreement. The obtained $\kappa_{\eta} /\left(k_{\mathrm{B}} T\right)$ from eq 13 is reported in Table 2 .

To examine the effect of different dynamics by a modelindependent approach, we have calculated the MSD, $\left\langle\Delta r(t)^{2}\right\rangle$, for the 1 wt $\% d-C_{18}-d$-PEO4-DOPC sample using eq 15 along with the non-Gaussian parameter, $\alpha_{2}$, as illustrated in Figure 
6(a,b), respectively. For a better illustration of changes, we included earlier on pure DOPC, which shows a $t^{0.26 \pm 0.03}$ power
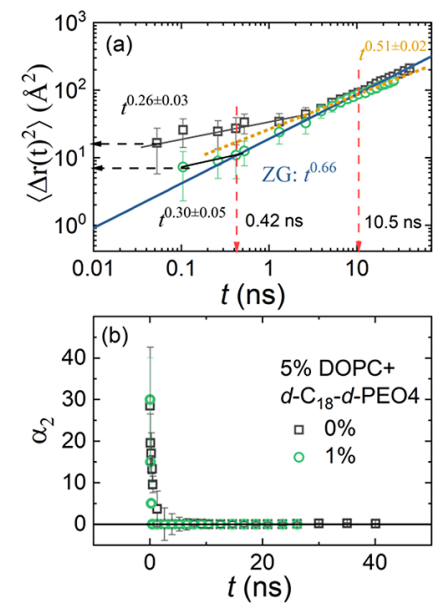

Figure 6. (a) Mean square displacement, $\left\langle\Delta r(t)^{2}\right\rangle$, vs Fourier time, $t$, for $5 \mathrm{wt} \% h$-DOPC ${ }^{41}$ and $5 \mathrm{wt} \% h$-DOPC dispersed in $1 \mathrm{wt} \% d-\mathrm{C}_{18^{-}}$ $d$-PEO4 in $\mathrm{D}_{2} \mathrm{O}$ at $20^{\circ} \mathrm{C}$. The solid lines represent the experimental power-law dependence. The horizontal dashed arrow indicates absolute values of $\left\langle\Delta r(t)^{2}\right\rangle$ for $t \leq 0.1$ ns. (b) Corresponding nonGaussian parameter $\alpha_{2}$.

law dependence at low Fourier times, $t<3$ ns, and finite $\alpha_{2}$ seems to be related to the tail motion. ${ }^{41,50}$ We will now discuss potential sources of these differences.

First, the ZG region ( $t^{0.66}$ region) stays almost constant, but the short-time region is highly affected. Such a disappearance was observed earlier in the case of contrast-matched lipid tails. Hence, we discuss the likelihood of contrast matching of the lipid tails first.

Contrast Matching. Three independent arguments deem contrast matching less likely. (i) A low concentration of the polymer in the bilayer. (ii) In case the tail is entirely contrast matched, there would be a visible contribution by the headgroups in $\Gamma_{\mathrm{q}} / Q^{3}$. (iii) The contrast conditions are far from the contrast-matching conditions.

(i) Our calculations (cf. SI) suggested that for 5 wt \% DOPC used in NSE the concentration range of the polymer in the outermost leaflet ranges from 0.02 to 0.08 wt $\%$. This concentration is too low to cause any visible effect on the contrast of the NSE experiment.

(ii) There is further support that the contrast may not be matched. In the case of lipid tail contrast matched with the solvent, the scattering of the lipid headgroup prevails, which allows the observation of membrane thickness fluctuations as a peak in the normalized ZG decay rate $\Gamma_{Q} / Q^{3}$ vs $Q$ plot. ${ }^{47,61}$ Figure $S 13$ in the SI illustrates $\Gamma_{Q} / Q^{3}=$ const. This suggests that the contribution of the headgroup does not prevail. The absence of the membrane fluctuations alone is not full proof that the tails are not contrast-matched. However, this experimental observation together with the absence of a peak $\Gamma_{q} / Q^{3}$ augments the confidence that the tail is not contrast-matched.

(iii) Finally, we ask what the contrast-matching condition would be for the lipid tail and hydrophobic block $d-\mathrm{C}_{18}$. The idea is to consider the hydrogenous lipid tails, the deuterated hydrophobic $d-C_{18}$, and an aqueous solution with $\mathrm{D}_{2} \mathrm{O}$ and $\mathrm{H}_{2} \mathrm{O}$ as a quaternary system in which the contrast is determined by the difference in the average scattering length densities of the $d / h$-solvent and the lipid tail $/ d-C_{18}$. Such a simplified consideration neglects the formation of domains or rafts, which will be discussed below. For such a system, the contrast is $\Delta \rho$ $=\bar{\rho}_{\text {tail } / d-\mathrm{C} 18}-\bar{\rho}_{d / h \text {-solvent. }}$ The average scattering-length densities are $\bar{\rho}_{\text {tail } / d-\mathrm{C} 18}=\phi_{1} \rho_{\text {tail }}+\left(1-\phi_{1}\right) \rho_{d-\mathrm{C}_{18}}$ and $\bar{\rho}_{d / h \text {-solvent }}=\phi_{2} \rho_{\mathrm{D}_{2} \mathrm{O}}+\left(1-\phi_{2}\right) \rho_{\mathrm{H}_{2} \mathrm{O}}$. The NSE experiments were conducted in $\mathrm{D}_{2} \mathrm{O}$, hence $\phi_{2}=1$ and $\bar{\rho}_{d / h \text {-solvent }}=\rho_{\mathrm{D}_{2} \mathrm{O}}$. The neutron scattering-length density of the tail is $\rho_{\text {tail }}=4.6 \times 10^{8} \mathrm{~cm}^{-2}$, and the hydrophobic part of $d$ - $\mathrm{C}_{18}-d$-PEO4 is $\rho_{d-C_{18}}=6.52 \times 10^{10}$ $\mathrm{cm}^{-2} \cdot{ }^{37,43,77}$ Contrast-matching conditions imply that $\Delta \rho=0$ or $\rho_{\mathrm{D}_{2} \mathrm{O}}=\tilde{\phi}_{1} \rho_{\text {tail }}+\left(1-\tilde{\phi}_{1}\right) \rho_{d-\mathrm{C}_{18}}$. Therefore, we can calculate the contrast-matching concentration from $\tilde{\phi}_{1}=\left(\rho_{\mathrm{D}_{2} \mathrm{O}}-\rho_{d-\mathrm{C}_{18}}\right) /\left(\rho_{\text {tail }}-\rho_{d-\mathrm{C}_{18}}\right)$. As shown in Figure S15 of the SI, we need a mixture of $98 \% d-C_{18}$ chains in $h$-DOPC lipid to contrast match with $\mathrm{D}_{2} \mathrm{O}$. In the samples used, the deuterated chain fraction in the bilayer is significantly lower than that.

Physical Meaning of the (Almost) Disappearance of the Short-Time Region. The MSD in Figure 6 illustrates the lowered contribution of the tail dynamics at low Fourier times to the MSD. It may entirely disappear, or the two values at the shortest Fourier times may indicate a small residue of the MSD of the tail motion. We use the fact that the Q-range of the NSE experiment essentially represents the length of the cylindrical confinement, as discussed in a previous publication. The summary of SANS results (Table 1) shows that the thickness of the bilayer changes only from $3.6 \pm 0.1$ to $3.4 \pm 0.2 \mathrm{~nm}$.

If we compare the mixture of DOPC with the polymer, then this is a situation similar to the case of DMPC/DOPC mixtures (i.e., mixtures of saturated and unsaturated hydrocarbons). In the case of adding DOPC to DMPC, the data seem to be in favor of stronger tail confinement. ${ }^{78}$ However, the low concentration of polymer inserted into the liposome observed by SANS ( 0.02 to 0.08 ) may not argue in favor of any dilution effect by adding a saturated hydrocarbon chain (polymer) to an unsaturated lipid vesicle.

The structural information together with the calculated number of polymers in the bilayer makes a change in the confinement less likely, at least in the time- and length-scale regions of the NSE experiment. Hence, we cannot reach a final conclusion, and further experiments will be necessary to understand what causes the disappearance of the short-term motion that was assigned to the tail earlier.

We can utilize the model-free approach to obtain the changes in the membrane rigidity in the presence of the polymer. In Figure 7 , the calculated $\kappa_{\eta} / k_{\mathrm{B}} T$ for a 1 wt $\% d-\mathrm{C}_{18^{-}}$ d-PEO4-DOPC sample for MLVs with $N=2$ is illustrated. An equivalent ULV membrane rigidity, $\kappa_{\eta}(\mathrm{ULV})=\frac{\kappa_{\eta}(\mathrm{MLV})}{N}$, is used for comparison with a 0 wt $\% d-\mathrm{C}_{18}-d$-PEO4-DOPC sample (ULV) from our previous work. ${ }^{41,50}$ In this approach, any deviation from the ZG model is magnified. The shaded area in Figure 7 elucidates a wider region (time-independent behavior) in the presence of $1 \mathrm{wt} \% d-\mathrm{C}_{18}-d$-PEO4 polymer that behaves like the standard ZG motion.

In the limit of $Q \rightarrow 0$, we have $\kappa_{\eta} / k_{\mathrm{B}} T \propto t^{2-3 x}$. In this case, the ZG prediction with $x=2 / 3=0.66$ yields time-independent behavior (solid lines), whereas the lipid tail motion has $x=$ 


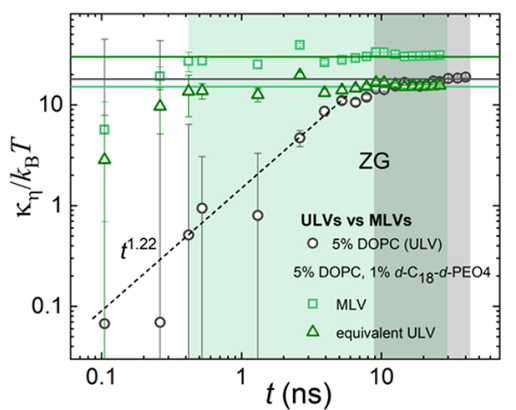

Figure 7. Membrane rigidity, $\kappa_{\eta}$, divided by the thermal energy, $k_{\mathrm{B}} T$, with the Boltzmann constant, $k_{\mathrm{B}}$, and the temperature, $T$, as a function of Fourier time. The data is calculated over the NSE time window from the MSD in Figure 6(a) for 0 and $1 \mathrm{wt} \% d-\mathrm{C}_{18}-d$-PEO4 polymer dispersed in $\mathrm{D}_{2} \mathrm{O}$ at $20{ }^{\circ} \mathrm{C}$. The calculated average values from the flat ZG region (shaded green and gray, respectively) are illustrated by the horizontal lines. These lines represent the bending modulus, $k / k_{\mathrm{B}} T$, and the values are listed in Table 2 . The different power laws are explained in the text.

0.26 , yielding a $t^{1.22}$ contribution (dotted line). This simple estimate assumes that the effect of translational diffusion of the vesicle is negligible $\left(D_{\mathrm{t}}=0\right)$, and $\alpha_{2}=0$. It should be noted that for a 1 wt $\% d$ - $\mathrm{C}_{18}$ - $d$-PEO4 sample the additional $t^{0.51}$ dependence in MSD analysis (Figure 6 (a)) for $t>10.5$ ns has been eliminated by subtracting a $t^{0.47}$ contribution following the $\kappa_{\eta} / k_{\mathrm{B}} T \propto t^{2-3 x}$ dependence, with $x=0.51$. This yields $\kappa_{\eta}$ / $k_{\mathrm{B}} T=16 \pm 2$ for the 1 wt $\% d-\mathrm{C}_{18}-d$-PEO4 sample.

In Table 2, we have compared $\kappa_{\eta} / k_{\mathrm{B}} T$ obtained from ZG (eq 10), multiplicative (eq 9), and MSD analysis (Figure 6) approaches using the $\mathrm{D}_{2} \mathrm{O}$ viscosity in eqs 12 and 13 . We have also included the calculation from the ZG model (equation) using only the higher Fourier times $(t>5 \mathrm{~ns})$ for the analysis, which results in $\kappa_{\eta} / k_{\mathrm{B}} T$ values similar to those obtained by the multiplicative and MSD analysis. This emphasizes that the undulations prevail in the intermediate time range. For a proper assessment of the single bilayer rigidity of the $1 \mathrm{wt} \% d$ $\mathrm{C}_{18}$-d-PEO4-DOPC sample, we have compared with the $0 \% d$ $\mathrm{C}_{18}$-d-PEO4-DOPC sample by calculating the equivalent ULV membrane rigidity, $\kappa_{\eta}(N=1)=\frac{\kappa_{\eta}(N=2)}{2}$. This analysis clearly elucidates the fact that in the presence of 1 wt $\% d-C_{18}-d$-PEO4 polymer the membrane rigidity in each bilayer decreases.

Several previous studies have explored phospholipid membrane rigidity changes upon interactions with structurally or chemically similar molecules. In a 2018 study, Elsayed et al. have shown that the membrane rigidity can be decreased with nonionic surfactants such as the ones discussed in the Introduction as well as $\mathrm{C}_{18: 1} \mathrm{EO}_{20}$ molecules which are structurally quite similar to the $n$-alkyl PEO polymer used in this work. ${ }^{79}$ Some have reported an increased bending rigidity upon interactions with block copolymers. For instance, poly(MPC-PPO-MPC) polymers have been shown to increase the phospholipid vesicle rigidity by weak dipole-dipole interactions with the zwitterions. ${ }^{80}$ Despite the changes in collective dynamics, there are no reported changes in the static structure of the vesicles as observed in this case. Another study by Kang et al. explored the use of amphiphilic triblock copolymers (PEO-PCL-PEO and PEO-PDMS-PEO) with phospholipid giant unilamellar vesicles where they observed increased membrane rigidity due to lateral coassembly. ${ }^{81}$ The diversity of different block copolymers therefore can impact the membrane rigidity differently, providing an excellent opportunity to manipulate this property to match different applications.

In summary, from both structure and dynamics, we have observed that a disruptive effect introduced by nonionic surfactant-like polymers causes a transformation from ULV to MLV structures. In short, the proposed mechanism of MLV formation has been illustrated in Figure 8. At first, the polymer chain prefers to slice into the bilayer membrane. This phenomenon causes structural defects in the lipid bilayers, as illustrated in Figure 8A, supported by the TEM images. This disruption provides an opportunity for bridge formation (Figure $8 \mathrm{~B}$ ) between individual vesicles that leads to the transport of free lipids, which acts as a nucleation site for the formation of a new bilayer, resulting in the formation of MLVs (Figure $8 \mathrm{C}$ ) determined by SANS. A similar mechanism of the induced multilamellar structure was observed by using cell-

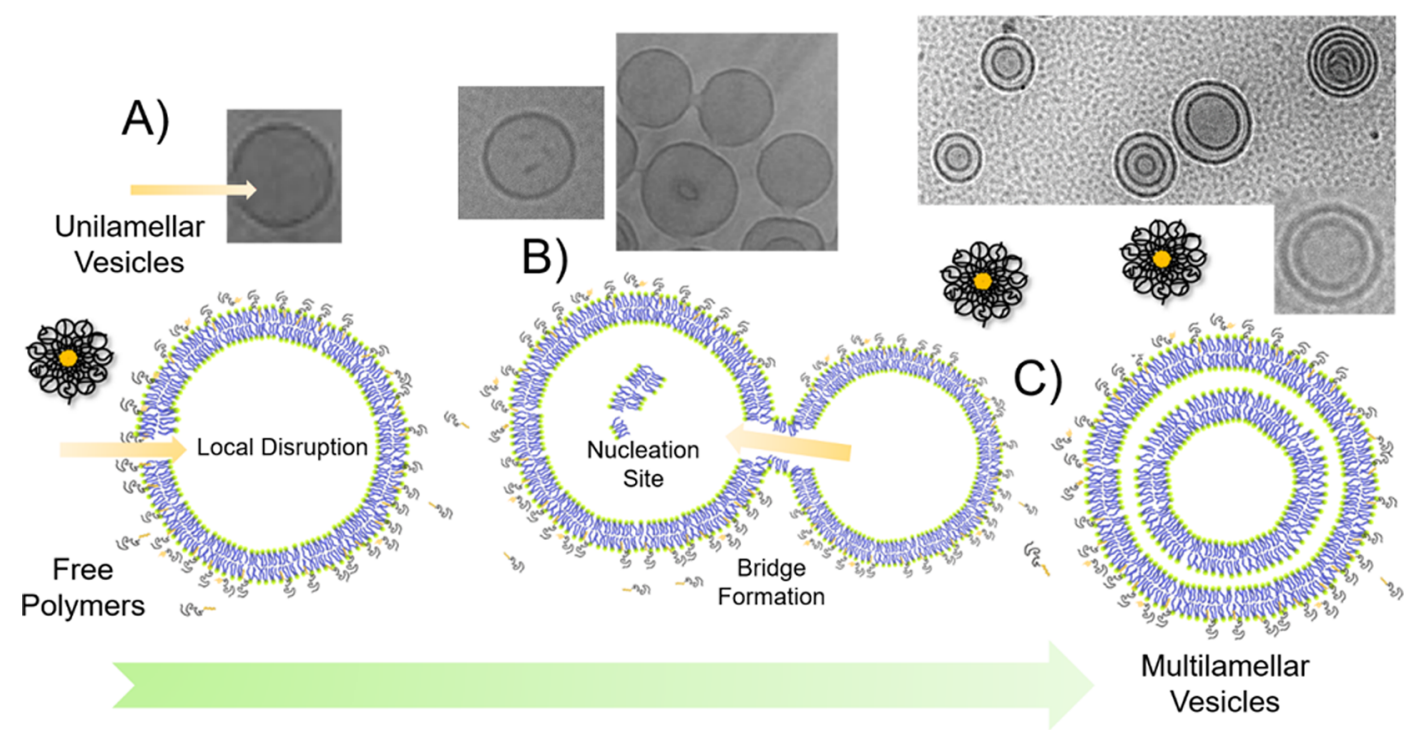

Figure 8. Schematic illustration of the proposed mechanism of MLV formation in (A) transient local disruption, (B) bridge formation, and (C) multilayer vesicles (MLVs) in the presence of $d-\mathrm{C}_{18}-d$-PEO4 polymers. 
penetrating peptides (CPPs) where the mechanism has transition steps involving similar transient pores. ${ }^{82}$ The presence of a free lipid layer as presented in the nucleation site has been observed in nanoemulsions. ${ }^{83}$ The reverse process (multi- to unilamellar transformation) has been observed in the case of lectin vesicles when subjected to diphenylalanine-based small peptides. The transition, in this case, occurs due to the formation of peptide aggregates on the membrane interface interacting with the phosphate groups. This destabilizes the fine balance of attractive repulsive forces in multilayered structures, resulting in the formation of larger unilamellar structures. ${ }^{84}$ Our present study is another example of a destabilization effect where a fine balance of these forces drives MLV formation.

\section{CONCLUSIONS}

Bringing together the information from SAXS and SANS, we can illustrate the effect of mixing $d-\mathrm{C}_{18}-d$-PEO4 and vesicle solutions in Figure 8. The polymer forms micelles in an aqueous solution as shown by Zinn et al. ${ }^{85}$ We observed from SAXS analysis that in the presence of lipid vesicles the polymer chain consisting of hydrophobic alkyl and hydrophilic PEO segments prefers to wedge into the bilayer membrane. The hydrophobic alkyl group of the polymer resides in the lipophilic hydrocarbon core of the lipid bilayer. Such preferential migration of the unimers into the membrane was also observed for bicontinuous microemulsions. ${ }^{86}$ The partial disruption of the bilayer lipid order by the polymer is responsible for about a $30 \%$ reduction in membrane rigidity in each bilayer, as determined by NSE. These polymers in the bilayer have their long hydrophilic PEO chains dangling outside the vesicle interface into the water. They exert a hydrophilic tension on the lipid membrane, causing an overall expansion in the size of the vesicles, as verified by DLS and SANS. The simultaneous formation of MLVs contributes further to the increase in the size of the vesicles. These results emphasize the opportunities to use a unique hydrophilichydrophobic polymer acting as a hybrid of diblock and nonionic surfactants that can transform membrane structures and control their dynamics with possible applications in topical drugs or nutraceutical formulations.

\section{ASSOCIATED CONTENT}

\section{(s) Supporting Information}

The Supporting Information is available free of charge at https://pubs.acs.org/doi/10.1021/acs.langmuir.0c03302.

Vesicle preparation, diffusion coefficients, cryo-TEM images, SAXS diffraction, NSE data on a linear scale, and ZG decay rate from NSE (PDF)

\section{AUTHOR INFORMATION}

\section{Corresponding Authors}

Judith U. De Mel - Department of Chemistry, Louisiana State University, Baton Rouge, Louisiana 70803, United States; 이이.org/0000-0001-7546-1491; Email: 1demel1@ lsu.edu

Sudipta Gupta - Department of Chemistry, Louisiana State University, Baton Rouge, Louisiana 70803, United States; ○ orcid.org/0000-0001-6642-3776; Email: g.sudipta26@ gmail.com

Gerald J. Schneider - Department of Chemistry and Department of Physics \& Astronomy, Louisiana State
University, Baton Rouge, Louisiana 70803, United States; ○ orcid.org/0000-0002-5577-9328; Email: gjschneider@ lsu.edu

\section{Authors}

Lutz Willner - Jülich Center for Neutron Science (JCNS-1) and Institute of Biological Information Processing (IBI-8) Forschungszentrum Jülich GmbH, 52428 Jülich, Germany

Jürgen Allgaier - Jülich Center for Neutron Science (JCNS-1) and Institute of Biological Information Processing (IBI-8) Forschungszentrum Jülich GmbH, 52428 Jülich, Germany; (1) orcid.org/0000-0002-9276-597X

Laura R. Stingaciu - Neutron Sciences Directorate, Oak Ridge National Laboratory (ORNL), Oak Ridge, Tennessee 37831, United States

Markus Bleuel - NIST Center for Neutron Research, National Institute of Standards and Technology, Gaithersburg, Maryland 20899-8562, United States

Complete contact information is available at:

https://pubs.acs.org/10.1021/acs.langmuir.0c03302

\section{Notes}

The authors declare no competing financial interest.

\section{ACKNOWLEDGMENTS}

The neutron-scattering work is supported by the U.S. Department of Energy (DOE) under EPSCoR grant no. DESC0012432 with additional support from the Louisiana Board of Regents. Access to the neutron spin echo spectrometer and small-angle scattering instruments was provided by the Center for High-Resolution Neutron Scattering, a partnership between the National Institute of Standards and Technology and the National Science Foundation under agreement no. DMR1508249. Research conducted at the Spallation Neutron Source (SNS) at Oak Ridge National Laboratory (ORNL) was sponsored by the Scientific User Facilities Division, Office of Basic Energy Sciences, U.S. DOE. We thank Lin Yang and Shirish Chodankar from the 16-ID, LIX beamline at National Synchrotron Light Source (NSLS) II. The LiX beamline is part of the Life Science Biomedical Technology Research resource, primarily supported by the National Institutes of Health, the National Institute of General Medical Sciences under grant P41 GM111244, and by the Department of Energy Office of Biological and Environmental Research under grant KP1605010, with additional support from NIH grant S10 OD012331. As an NSLS II facility resource at Brookhaven National Laboratory, work performed at Life Science and Biomedical Technology Research is supported in part by the U.S. Department of Energy, Office of Basic Energy Sciences Program under contract DE-SC0012704. We thank Thomas Weiss from BL 4-2 at the Stanford Synchrotron Radiation Lightsource for assisting with SAXS experiments. Use of the Stanford Synchrotron Radiation Lightsource, SLAC National Accelerator Laboratory, is supported by the U.S. Department of Energy, Office of Science, Office of Basic Energy Sciences under contract no. DE-AC02-76SF00515. The SSRL Structural Molecular Biology Program is supported by the DOE Office of Biological and Environmental Research and by the National Institutes of Health, National Institute of General Medical Sciences (including P41GM103393). The contents of this publication are solely the responsibility of the authors and do not necessarily represent the official views of NIGMS or NIH. We acknowledge Gina Guillory (past undergraduate research- 
er) for assisting with preliminary experiments. We also specially acknowledge Jiabo $\mathrm{He}$ at Tulane University for assisting with cryo-TEM experiments. Certain trade names and company products are identified in order to specify adequately the experimental procedure. In no case does such identification imply recommendation or endorsement by the National Institute of Standards and Technology nor does it imply that the products are necessarily the best for the purpose. This report was prepared as an account of work sponsored by an agency of the United States Government. Neither the United States Government, nor any agency thereof, nor any of their employees make any warranty, express or implied, or assume any legal liability or responsibility for the accuracy, completeness, or usefulness of any information, apparatus, product, or process disclosed or represent that its use would not infringe on privately owned rights. Reference herein to any specific commercial product, process, or service by trade name, trademark, manufacturer, or otherwise does not necessarily constitute or imply its endorsement, recommendation, or favoring by the United States government or any agency thereof. The views and opinions of the authors expressed herein do not necessarily state or reflect those of the United States government or any agency thereof.

\section{REFERENCES}

(1) Verma, D. D.; Verma, S.; Blume, G.; Fahr, A. Particle size of liposomes influences dermal delivery of substances into skin. Int. J. Pharm. 2003, 258 (1), 141-151.

(2) Moghimi, S. M. The effect of methoxy-PEG chain length and molecular architecture on lymph node targeting of immuno-PEG liposomes. Biomaterials 2006, 27 (1), 136-144.

(3) Bulbake, U.; Doppalapudi, S.; Kommineni, N.; Khan, W. Liposomal Formulations in Clinical Use: An Updated Review. Pharmaceutics 2017, 9 (12), 1-33.

(4) Kim, M.; Vala, M.; Ertsgaard, C. T.; Oh, S.-H.; Lodge, T. P.; Bates, F. S.; Hackel, B. J. Surface Plasmon Resonance Study of the Binding of PEO-PPO-PEO Triblock Copolymer and PEO Homopolymer to Supported Lipid Bilayers. Langmuir 2018, 34 (23), 67036712.

(5) Zhao, Z.; Ji, X.; Dimova, R.; Lipowsky, R.; Liu, Y. Viscoelasticity of Poly(ethylene glycol) Solutions on Supported Lipid Bilayers via Quartz Crystal Microbalance with Dissipation. Macromolecules 2015, 48 (6), 1824-1831.

(6) Tirosh, O.; Barenholz, Y.; Katzhendler, J.; Priev, A. Hydration of polyethylene glycol-grafted liposomes. Biophys. J. 1998, 74 (3), 13719.

(7) Silvander, M.; Hansson, P.; Edwards, K. Liposomal Surface Potential and Bilayer Packing As Affected by PEG-Lipid Inclusion. Langmuir 2000, 16 (8), 3696-3702.

(8) Firestone, M. A.; Seifert, S. Interaction of Nonionic PEO-PPO Diblock Copolymers with Lipid Bilayers. Biomacromolecules 2005, 6 (5), 2678-2687.

(9) Firestone, M. A.; Wolf, A. C.; Seifert, S. Small-Angle X-ray Scattering Study of the Interaction of Poly(ethylene oxide)-bPoly(propylene oxide)-b-Poly(ethylene oxide) Triblock Copolymers with Lipid Bilayers. Biomacromolecules 2003, 4 (6), 1539-1549.

(10) Liu, G.; Fu, L.; Zhang, G. Role of Hydrophobic Interactions in the Adsorption of Poly(ethylene glycol) Chains on Phospholipid Membranes Investigated with a Quartz Crystal Microbalance. J. Phys. Chem. B 2009, 113 (11), 3365-3369.

(11) Gutberlet, T.; Kiselev, M.; Heerklotz, H.; Klose, G. SANS study of mixed POPC/C12En aggregates. Phys. B 2000, 276-278, 381383.

(12) Rieber, K.; Sýkora, J.; Olżyńska, A.; Jelinek, R.; Cevc, G.; Hof, $\mathrm{M}$. The use of solvent relaxation technique to investigate headgroup hydration and protein binding of simple and mixed phosphatidylcho- line/surfactant bilayer membranes. Biochim. Biophys. Acta, Biomembr. 2007, 1768 (5), 1050-1058.

(13) Chimisso, V.; Maffeis, V.; Hürlimann, D.; Palivan, C. G.; Meier, W. Self-Assembled Polymeric Membranes and Nanoassemblies on Surfaces: Preparation, Characterization, and Current Applications. Macromol. Biosci. 2020, 20 (1), 1900257.

(14) Schulz, M.; Olubummo, A.; Binder, W. H. Beyond the lipidbilayer: interaction of polymers and nanoparticles with membranes. Soft Matter 2012, 8 (18), 4849-4864.

(15) Zaki, A. M.; Carbone, P. How the Incorporation of Pluronic Block Copolymers Modulates the Response of Lipid Membranes to Mechanical Stress. Langmuir 2017, 33 (46), 13284-13294.

(16) Chen, D.; Santore, M. M. Hybrid copolymer-phospholipid vesicles: phase separation resembling mixed phospholipid lamellae, but with mechanical stability and control. Soft Matter 2015, 11 (13), 2617-2626.

(17) Mineart, K. P.; Venkataraman, S.; Yang, Y. Y.; Hedrick, J. L.; Prabhu, V. M. Fabrication and Characterization of Hybrid Stealth Liposomes. Macromolecules 2018, 51 (8), 3184-3192.

(18) Lasic, D. D. Sterically Stabilized Vesicles. Angew. Chem., Int. Ed. Engl. 1994, 33 (17), 1685-1698.

(19) Gupta, S.; Biehl, R.; Sill, C.; Allgaier, J.; Sharp, M.; Ohl, M.; Richter, D. Protein Entrapment in Polymeric Mesh: Diffusion in Crowded Environment with Fast Process on Short Scales. Macromolecules 2016, 49 (5), 1941-1949.

(20) Xuan, S.; Gupta, S.; Li, X.; Bleuel, M.; Schneider, G. J.; Zhang, D. Synthesis and Characterization of Well-Defined PEGylated Polypeptoids as Protein-Resistant Polymers. Biomacromolecules 2017, 18 (3), 951-964.

(21) Tribet, C.; Vial, F. Flexible macromolecules attached to lipid bilayers: impact on fluidity, curvature, permeability and stability of the membranes. Soft Matter 2008, 4 (1), 68-81.

(22) Kim, M.; Heinrich, F.; Haugstad, G.; Yu, G.; Yuan, G.; Satija, S. K.; Zhang, W.; Seo, H. S.; Metzger, J. M.; Azarin, S. M.; Lodge, T. P.; Hackel, B. J.; Bates, F. S. Spatial Distribution of PEO-PPO-PEO Block Copolymer and PEO Homopolymer in Lipid Bilayers. Langmuir 2020, 36 (13), 3393-3403.

(23) Senac, C.; Urbach, W.; Kurtisovski, E.; Hünenberger, P. H.; Horta, B. A. C.; Taulier, N.; Fuchs, P. F. J. Simulating Bilayers of Nonionic Surfactants with the GROMOS-Compatible 2016H66 Force Field. Langmuir 2017, 33 (39), 10225-10238.

(24) Dong, R.; Hao, J. Complex Fluids of Poly(oxyethylene) Monoalkyl Ether Nonionic Surfactants. Chem. Rev. 2010, 110 (9), $4978-5022$.

(25) Sommer, C.; Pedersen, J. S.; Garamus, V. M. Structure and Interactions of Block Copolymer Micelles of Brij 700 Studied by Combining Small-Angle X-ray and Neutron Scattering. Langmuir 2005, 21 (6), 2137-2149.

(26) Zhao, W.; Uehera, S.; Tanaka, K.; Tadokoro, S.; Kusamori, K.; Katsumi, H.; Sakane, T.; Yamamoto, A. Effects of Polyoxyethylene Alkyl Ethers on the Intestinal Transport and Absorption of Rhodamine 123: A P-glycoprotein Substrate by In Vitro and In Vivo Studies. J. Pharm. Sci. 2016, 105 (4), 1526-34.

(27) Tang, J.; Wang, Y.; Wang, D.; Wang, Y.; Xu, Z.; Racette, K.; Liu, F. Key Structure of Brij for Overcoming Multidrug Resistance in Cancer. Biomacromolecules 2013, 14 (2), 424-430.

(28) Zhang, W.; Metzger, J. M.; Hackel, B. J.; Bates, F. S.; Lodge, T. $P$. Influence of the Headgroup on the Interaction of Poly(ethylene oxide)-Poly(propylene oxide) Block Copolymers with Lipid Bilayers. J. Phys. Chem. B 2020, 124 (12), 2417-2424.

(29) Johnsson, M.; Edwards, K. Interactions between Nonionic Surfactants and Sterically Stabilized Phophatidyl Choline Liposomes. Langmuir 2000, 16 (23), 8632-8642.

(30) De La Maza, A.; Parra, J. L.; Garcia, M. T.; Ribosa, I.; Leal, J. S. Permeability changes in the phospholipid bilayer caused by nonionic surfactants. J. Colloid Interface Sci. 1992, 148 (2), 310-316.

(31) Sujatha, J.; Mishra, A. K. Effect of ionic and neutral surfactants on the properties of phospholipid vesicles: investigation using 
fluorescent probes. J. Photochem. Photobiol., A 1997, 104 (1), 173178.

(32) Van Zutphen, H.; Merola, A. J.; Brierley, G. P.; Cornwell, D. G. The interaction of nonionic detergents with lipid bilayer membranes. Arch. Biochem. Biophys. 1972, 152 (2), 755-766.

(33) Liu, G.; Zhang, G. Interactions between Polymers and Phospholipid Membranes. In QCM-D Studies on Polymer Behavior at Interfaces; Liu, G., Zhang, G., Eds.; Springer: Berlin, 2013; pp 71-81.

(34) Venturoli, M.; Smit, B.; Sperotto, M. M. Simulation Studies of Protein-Induced Bilayer Deformations, and Lipid-Induced Protein Tilting, on a Mesoscopic Model for Lipid Bilayers with Embedded Proteins. Biophys. J. 2005, 88 (3), 1778-1798.

(35) Harzer, U.; Bechinger, B. Alignment of lysine-anchored membrane peptides under conditions of hydrophobic mismatch: a $\mathrm{CD}, 15 \mathrm{~N}$ and $31 \mathrm{P}$ solid-state NMR spectroscopy investigation. Biochemistry 2000, 39 (43), 13106-14.

(36) Silvestre, M. P. C.; Chaiyasit, W.; Brannan, R. G.; McClements, D. J.; Decker, E. A. Ability of Surfactant Headgroup Size To Alter Lipid and Antioxidant Oxidation in Oil-in-Water Emulsions. J. Agric. Food Chem. 2000, 48 (6), 2057-2061.

(37) Zinn, T.; Willner, L.; Lund, R.; Pipich, V.; Appavou, M. S.; Richter, D. Surfactant or block copolymer micelles? Structural properties of a series of well-defined n-alkyl-PEO micelles in water studied by SANS. Soft Matter 2014, 10 (28), 5212-20.

(38) Gupta, S.; Bleuel, M.; Schneider, G. J. A new ultrasonic transducer sample cell for in situ small-angle scattering experiments. Rev. Sci. Instrum. 2018, 89 (1), 015111.

(39) Ulrich, A. S.; Sami, M.; Watts, A. Hydration of DOPC bilayers by differential scanning calorimetry. Biochim. Biophys. Acta, Biomembr. 1994, 1191 (1), 225-230.

(40) Gupta, S.; Camargo, M.; Stellbrink, J.; Allgaier, J.; Radulescu, A.; Lindner, P.; Zaccarelli, E.; Likos, C. N.; Richter, D. Dynamic phase diagram of soft nanocolloids. Nanoscale 2015, 7 (33), 13924-34.

(41) Gupta, S.; De Mel, J. U.; Perera, R. M.; Zolnierczuk, P.; Bleuel, M.; Faraone, A.; Schneider, G. J. Dynamics of Phospholipid Membranes beyond Thermal Undulations. J. Phys. Chem. Lett. 2018, 9, 2956-2960.

(42) Sternhagen, G. L.; Gupta, S.; Zhang, Y.; John, V.; Schneider, G. J.; Zhang, D. Solution Self-Assemblies of Sequence-Defined Ionic Peptoid Block Copolymers. J. Am. Chem. Soc. 2018, 140 (11), 41004109.

(43) Hoffmann, I.; Michel, R.; Sharp, M.; Holderer, O.; Appavou, M. S.; Polzer, F.; Farago, B.; Gradzielski, M. Softening of phospholipid membranes by the adhesion of silica nanoparticles - as seen by neutron spin-echo (NSE). Nanoscale 2014, 6 (12), 6945-52.

(44) Nallet, F.; Laversanne, R.; Roux, D. Modelling X-ray or Neutron Scattering Spectra of Lyotropic Lamellar Phases: Interplay Between Form and Structure Factors. J. Phys. II 1993, 3, 487-502.

(45) Berghausen, J.; Zipfel, J.; Lindner, P.; Richtering, W. Influence of Water-Soluble Polymers on the Shear-Induced Structure Formation in Lyotropic Lamellar Phases. J. Phys. Chem. B 2001, 105 (45), 11081-11088.

(46) De Mel, J. U.; Gupta, S.; Perera, R. M.; Ngo, L. T.; Zolnierczuk, P.; Bleuel, M.; Pingali, S. V.; Schneider, G. J. Influence of external $\mathrm{NaCl}$ salt on membrane rigidity of neutral DOPC vesicles. Langmuir 2020, 36, 9356-9367.

(47) Woodka, A. C.; Butler, P. D.; Porcar, L.; Farago, B.; Nagao, M. Lipid bilayers and membrane dynamics: insight into thickness fluctuations. Phys. Rev. Lett. 2012, 109 (5), 058102.

(48) Hoffmann, I.; Michel, R.; Sharp, M.; Holderer, O.; Appavou, M. S.; Polzer, F.; Farago, B.; Gradzielski, M. Softening of phospholipid membranes by the adhesion of silica nanoparticles-as seen by neutron spin-echo (NSE). Nanoscale 2014, 6 (12), 6945-52.

(49) Nickels, J. D.; Cheng, X.; Mostofian, B.; Stanley, C.; Lindner, B.; Heberle, F. A.; Perticaroli, S.; Feygenson, M.; Egami, T.; Standaert, R. F.; Smith, J. C.; Myles, D. A.; Ohl, M.; Katsaras, J. Mechanical Properties of Nanoscopic Lipid Domains. J. Am. Chem. Soc. 2015, 137 (50), 15772-80.
(50) Gupta, S.; Schneider, G. J. Modeling the Dynamics of Phospholipids in the Fluid Phase of Liposomes. Soft Matter 2020, 16, 3245-3256.

(51) Zilman, A. G.; Granek, R. Undulations and Dynamic Structure Factor of Membranes. Phys. Rev. Lett. 1996, 77 (23), 4788-4791.

(52) Watson, M. C.; Brown, F. L. Interpreting membrane scattering experiments at the mesoscale: the contribution of dissipation within the bilayer. Biophys. J. 2010, 98 (6), L9-L11.

(53) Sharma, V. K.; Hayes, D. G.; Gupta, S.; Urban, V. S.; O’Neill, H. M.; Pingali, S. V.; Ohl, M.; Mamontov, E. Incorporation of Melittin Enhances Interfacial Fluidity of Bicontinuous Microemulsions. J. Phys. Chem. C 2019, 123 (17), 11197-11206.

(54) Boal, D. Mechanics of the Cell; Cambridge University Press: Cambridge, U.K., 2002; Vol. 2, p 267.

(55) Nagao, M.; Kelley, E. G.; Ashkar, R.; Bradbury, R.; Butler, P. D. Probing Elastic and Viscous Properties of Phospholipid Bilayers Using Neutron Spin Echo Spectroscopy. J. Phys. Chem. Lett. 2017, 8, 46794684.

(56) Rand, R. P.; Fuller, N. L. Structural dimensions and their changes in a reentrant hexagonal-lamellar transition of phospholipids. Biophys. J. 1994, 66 (6), 2127-2138.

(57) Templer, R. H.; Khoo, B. J.; Seddon, J. M. Gaussian Curvature Modulus of an Amphiphilic Monolayer. Langmuir 1998, 14 (26), $7427-7434$.

(58) Winterhalter, M.; Helfrich, W. Bending elasticity of electrically charged bilayers: coupled monolayers, neutral surfaces, and balancing stresses. J. Phys. Chem. 1992, 96 (1), 327-330.

(59) Campelo, F.; Arnarez, C.; Marrink, S. J.; Kozlov, M. M. Helfrich model of membrane bending: from Gibbs theory of liquid interfaces to membranes as thick anisotropic elastic layers. Adv. Colloid Interface Sci. 2014, 208, 25-33.

(60) Kollmitzer, B.; Heftberger, P.; Rappolt, M.; Pabst, G. Monolayer spontaneous curvature of raft-forming membrane lipids. Soft Matter 2013, 9 (45), 10877-10884.

(61) Gupta, S.; De Mel, J. U.; Schneider, G. J. Dynamics of liposomes in the fluid phase. Curr. Opin. Colloid Interface Sci. 2019, $42,121-136$

(62) Gerstl, C.; Schneider, G. J.; Fuxman, A.; Zamponi, M.; Frick, B.; Seydel, T.; Koza, M.; Genix, A. C.; Allgaier, J.; Richter, D.; Colmenero, J.; Arbe, A. Quasielastic Neutron Scattering Study on the Dynamics of Poly(alkylene oxide)s. Macromolecules 2012, 45 (10), 4394-4405.

(63) Schneider, G. J.; Nusser, K.; Neueder, S.; Brodeck, M.; Willner, L.; Farago, B.; Holderer, O.; Briels, W. J.; Richter, D. Anomalous chain diffusion in unentangled model polymer nanocomposites. Soft Matter 2013, 9 (16), 4336-4348.

(64) Jeon, J.-H.; Javanainen, M.; Martinez-Seara, H.; Metzler, R.; Vattulainen, I. Protein Crowding in Lipid Bilayers Gives Rise to NonGaussian Anomalous Lateral Diffusion of Phospholipids and Proteins. Phys. Rev. X 2016, 6 (2), 021006.

(65) Glinka, C. J.; Barker, J. G.; Hammouda, B.; Krueger, S.; Moyer, J. J.; Orts, W. J. The $30 \mathrm{~m}$ Small-Angle Neutron Scattering Instruments at the National Institute of Standards and Technology. J. Appl. Crystallogr. 1998, 31 (3), 430-445.

(66) Choi, S. M.; Barker, J. G.; Glinka, C. J.; Cheng, Y. T.; Gammel, P. L. Focusing cold neutrons with multiple biconcave lenses for smallangle neutron scattering. J. Appl. Crystallogr. 2000, 33 (3), 793-796.

(67) Kline, S. R. Reduction and analysis of SANS and USANS data using IGOR Pro. J. Appl. Crystallogr. 2006, 39 (6), 895-900.

(68) Glatter, O.; Kratky, O. Small Angle X-ray Scattering; Academic Press: London, 1982.

(69) Lindner, P.; Zemb, Th. Neutron, X-rays and Light: Scattering Methods Applied to Soft Condensed Matter; Elsevier Science: Amsterdam, 2002.

(70) Ohl, M.; Monkenbusch, M.; Arend, N.; Kozielewski, T.; Vehres, G.; Tiemann, C.; Butzek, M.; Soltner, H.; Giesen, U.; Achten, R.; Stelzer, H.; Lindenau, B.; Budwig, A.; Kleines, H.; Drochner, M.; Kaemmerling, P.; Wagener, M.; Möller, R.; Iverson, E. B.; Sharp, M.; Richter, D. The spin-echo spectrometer at the Spallation Neutron 
Source (SNS). Nucl. Instrum. Methods Phys. Res., Sect. A 2012, 696, 85-99.

(71) Teixeira, J. Small-Angle Scattering by Fractal Systems. J. Appl. Crystallogr. 1988, 21, 781-785.

(72) Mildner, D. F. R.; Hall, P. L. Small-angle scattering from porous solids with fractal geometry. J. Phys. D: Appl. Phys. 1986, 19 (8), $1535-1545$.

(73) Hait, S. K.; Moulik, S. P. Determination of critical micelle concentration (CMC) of nonionic surfactants by donor-acceptor interaction with lodine and correlation of CMC with hydrophilelipophile balance and other parameters of the surfactants. J. Surfactants Deterg. 2001, 4 (3), 303-309.

(74) Courbin, L.; Delville, J. P.; Rouch, J.; Panizza, P. Instability of a Lamellar Phase under Shear Flow: Formation of Multilamellar Vesicles. Phys. Rev. Lett. 2002, 89 (14), 148305.

(75) Scott, H. L.; Skinkle, A.; Kelley, E. G.; Waxham, M. N.; Levental, I.; Heberle, F. A. On the Mechanism of Bilayer Separation by Extrusion, or Why Your LUVs Are Not Really Unilamellar. Biophys. J. 2019, 117 (8), 1381-1386.

(76) Kucerka, N.; Tristram-Nagle, S.; Nagle, J. F. Structure of fully hydrated fluid phase lipid bilayers with monounsaturated chains. J. Membr. Biol. 2006, 208 (3), 193-202.

(77) Benedetto, A.; Heinrich, F.; Gonzalez, M. A.; Fragneto, G.; Watkins, E.; Ballone, P. Structure and Stability of Phospholipid Bilayers Hydrated by a Room-Temperature Ionic Liquid/Water Solution: A Neutron Reflectometry Study. J. Phys. Chem. B 2014, 118 (42), 12192-12206.

(78) Nanda, H.; García Sakai, V.; Khodadadi, S.; Tyagi, M. S.; Schwalbach, E. J.; Curtis, J. E. Relaxation dynamics of saturated and unsaturated oriented lipid bilayers. Soft Matter 2018, 14 (29), 61196127.

(79) Elsayed, M. M. A.; Ibrahim, M. M.; Cevc, G. The effect of membrane softeners on rigidity of lipid vesicle bilayers: Derivation from vesicle size changes. Chem. Phys. Lipids 2018, 210, 98-108.

(80) Wang, J.-Y.; Chen, W.; Nagao, M.; Shelat, P.; Hammer, B. A. G.; Tietjen, G. T.; Cao, K. D.; Henderson, J. M.; He, L.; Lin, B.; Akgun, B.; Meron, M.; Qian, S.; Ward, S.; Marks, J. D.; Emrick, T.; Lee, K. Y. C. Tailoring Biomimetic Phosphorylcholine-Containing Block Copolymers as Membrane-Targeting Cellular Rescue Agents. Biomacromolecules 2019, 20 (9), 3385-3391.

(81) Kang, J. Y.; Choi, I.; Seo, M.; Lee, J. Y.; Hong, S.; Gong, G.; Shin, S. S.; Lee, Y.; Kim, J. W. Enhancing membrane modulus of giant unilamellar lipid vesicles by lateral co-assembly of amphiphilic triblock copolymers. J. Colloid Interface Sci. 2020, 561, 318-326.

(82) Allolio, C.; Magarkar, A.; Jurkiewicz, P.; Baxova, K.; Javanainen, M.; Mason, P. E.; Sachl, R.; Cebecauer, M.; Hof, M.; Horinek, D.; Heinz, V.; Rachel, R.; Ziegler, C. M.; Schrofel, A.; Jungwirth, P. Arginine-rich cell-penetrating peptides induce membrane multilamellarity and subsequently enter via formation of a fusion pore. Proc. Natl. Acad. Sci. U. S. A. 2018, 115 (47), 11923-11928.

(83) Sommerling, J. H.; de Matos, M. B. C.; Hildebrandt, E.; Dessy, A.; Kok, R. J.; Nirschl, H.; Leneweit, G. Instability Mechanisms of Water-in-Oil Nanoemulsions with Phospholipids: Temporal and Morphological Structures. Langmuir 2018, 34 (2), 572-584.

(84) Gerbelli, B. B.; da Silva, E. R.; Miranda Soares, B.; Alves, W. A.; Andreoli de Oliveira, E. Multilamellar-to-Unilamellar Transition Induced by Diphenylalanine in Lipid Vesicles. Langmuir 2018, 34 (5), 2171-2179.

(85) Zinn, T.; Willner, L.; Lund, R.; Pipich, V.; Richter, D. Equilibrium exchange kinetics in n-alkyl-PEO polymeric micelles: single exponential relaxation and chain length dependence. Soft Matter 2012, 8 (3), 623-626.

(86) Brodeck, M.; Maccarrone, S.; Saha, D.; Willner, L.; Allgaier, J.; Mangiapia, G.; Frielinghaus, H.; Holderer, O.; Faraone, A.; Richter, D. Asymmetric polymers in bicontinuous microemulsions and their accretion to the bending of the membrane. Colloid Polym. Sci. 2015, 293 (4), 1253-1265. 